\title{
Examining the energy loss in systems of stacked rocking bodies
}

\author{
M. N. Chatzis ${ }^{1}$, M. García Espinosa ${ }^{2}$, C. Needham ${ }^{3}$, and M. S. \\ Williams ${ }^{4}$
}

\begin{abstract}
Free-standing bodies, which rock in response to dynamic base excitations, can be found in a wide range of applications. There are many instances, for example a museum artifact seated on a support pedestal, where both the object and its support are freestanding, resulting in a dynamic system where both bodies can rock with respect to their contact interfaces. This paper analyses the rich dynamics of systems of two stacked rocking bodies. It focuses on the location of the resultant impulses between bodies during impact, considering their effect on the overall stability of the system. It is shown that the widely-used assumption that the impulses occur at the future rocking corners is the least conservative scenario and will often lead to underestimate of the likelihood of failure. The optimum configuration of two stacked blocks is found to be highly sensitive to the characteristics of the input motion and to the impulses location.
\end{abstract}

\section{INTRODUCTION}

The interest of the engineering community over the response of free-standing bodies subjected to vibrations has increased over the recent years. This is partly due to the realization that non-structural elements that are often free-standing are equally important to their structural counterparts (FEMA, 2006), and to an extent to a novel design philosophy that investigates the benefits of the lack of a full shear and tensile connection to the stability of structural elements. The fact that the shear connection of such bodies with their interface is achieved mainly through friction leads to a rocking response. This dynamic response becomes increasingly rich as more rigid modes are enabled, as when having assemblies of rocking bodies. The latter is a case which is met in several situations, as for example is the case of stacked rocking bodies found in museums composing of artifacts on pedestals. Assemblies of two stacked rocking bodies can be found as designs in several seismically active regions and hence their behaviour when subjected to ground motions is of interest.

The first dynamic model for the rocking response of a single body, the Inverted Pendulum Model (IPM), was presented by Housner (Housner, 1963) in 1963. In this model the response of a rocking body is simulated using a set of equivalent inverted pendulums. For this modelling approach, several assumptions must be introduced: the body and the base are considered to be rigid, sliding and uplifting

\footnotetext{
${ }^{1}$ Associate Professor in Dept. of Engineering Science, University of Oxford, United Kingdom, manolis.chatzis@eng.ox.ac.uk

${ }^{2} \mathrm{Ph} . \mathrm{D}$ Candidate in Dept. of Engineering Science, University of Oxford, United Kingdom, maria.garciaespinosa@eng.ox.ac.uk

${ }^{3}$ Innovation Product Manager, Shell, United Kingdom, christopher.needham@shell.com

${ }^{4}$ Professor in Department of Engineering Science, University of Oxford, United Kingdom, martin.williams@eng.ox.ac.uk
} 
are neglected, the response is planar and the loss of energy, which only occurs during impacts, is exclusively described by the dimensions of the body.

The work of Housner has been extended to rocking models for the dynamic behaviour of the assembly of two stacked rigid bodies. The associated conditions for failure of such systems have been studied in (Allen et al., 1986), while a complete formulation of the non-linear equations of motion using Newton's laws or Lagrange's method have been developed in (Psycharis, 1990) and (Spanos et al., 2001), respectively. Both focused on the derivation of the equation of motion for the consecutive transition from one pattern of motion to another making use of the assumption introduced by Housner: that of conserving angular momentum with respect to the future rocking corner. A multi-block model was developed in (Kounadis et al., 2012) where the simplified assumption of no loss of energy during impacts for each body in the system was adopted. Other type of rocking systems have been presented for rocking frames in (Makris and Vassiliou, 2014; Dimitrakopoulos and Giouvanidis, 2015), for rocking bodies on base isolation in (Harvey Jr, 2017; Vassiliou and Makris, 2012), for rocking bodies with casters in (Chatzis and Smyth, 2012a; Nikfar and Konstantinidis, 2017) and for a statuepedestal system in (Wittich and Hutchinson, 2016).

While a series of papers has relaxed several of the assumptions of the IPM (Psycharis and Jennings, 1983; Spanos et al., 2001; Palmeri and Makris, 2008; Zulli et al., 2012; Chatzis and Smyth, 2012c,b), the popularity of the IPM lies in its simplicity. It is further often claimed that the effects of sliding, uplift, ground and body deformability and three dimensional response can be minimised if the properties of the rocking body, support and interface are carefully designed. Even in such cases though, there is an inherent assumption of the IPM which would not be guaranteed to be satisfied, that of conserving angular momentum with respect to the future rocking corner and the resulting loss of energy during impacts (Chatzis et al., 2016).

Existing works in the literature (Zhang et al., 2012; Yim et al., 1980; Voyagaki et al., 2014; ElGawady et al., 2011) have used alternative assumptions about the loss of energy during impacts through the use of alternative values for the reduction of energy factor. A detailed discussion about the allowable energy loss of a rocking body modelled by the IPM is presented in (Chatzis et al., 2016). The authors explain that the vertical impulse during an impact may be applied in any location between the future rocking corner and the midpoint of the base, without violating the rest of the assumptions related to the $I P M$. This location is hence considered as a random variable with a related uncertainty, whose influence on the survival of the rocking body is studied. The paper emphasises that this assumption, the effect of which may not be minimised unless the contact interface is altered or the contact interface properties are taken into account in the model as in (Chatzis and Smyth, 2012c,b), has an important effect.

This work will hence extend the work presented in (Chatzis et al., 2016) to the case of stacked bodies. In particular, the paper will investigate the potential location of the vertical impulses at the interfaces between the two bodies and be- 
tween the lower body and the ground for the different types of impacts occurring in this system. The effect of this parameter on the overall stability of the top body and the system will be investigated.

\section{MODEL AND ASSUMPTIONS}

\section{Inverted Pendulum model for a single block.}

This analysis is restricted to planar, rectangular blocks rocking about either of their bottom corners without sliding and with at least one point always in contact with the ground. The geometry and the possible rocking patterns are defined in Figure 1. The block has width $2 b$, height $2 h$, mass $m$ and its moment of inertia about either 0 or $0^{\prime}$ is $I_{0}$. The distance from a corner to the centre of mass is $R=\sqrt{b^{2}+h^{2}}$ and the slenderness of the block is expressed by the angle $\alpha=\arctan \frac{b}{h}$. Dynamic excitation is in the form of a base acceleration with horizontal component $\ddot{x}_{g}$ and vertical component $\ddot{y}_{g}$.

[FIG. 1 about here.]

The possible motions of the block can be defined by three patterns: in pattern A rocking takes place about $0^{\prime}$ and the angle $\theta<0$, in pattern $\mathrm{B}$ rocking occurs about $0(\theta>0)$ and in pattern $\mathrm{C}$ there is no relative motion between the block and the base $(\theta=\dot{\theta}=0)$. (Housner, 1963) recognised that patterns $\mathrm{A}$ and $\mathrm{B}$ can be represented as inverted pendulums of length $R$, with a pin at the rocking corner, as in Figure 1. Application of Newton's second law to these pendulums gives the equations of motion:

$$
\begin{gathered}
\ddot{\theta}=-p^{2} \sin (\alpha-\theta)+\frac{m \ddot{x}_{g}}{I_{0}} R \cos (\alpha-\theta)-\frac{m \ddot{y}_{g}}{I_{0}} R \sin (\alpha-\theta) \text { for } \theta>0 \\
\ddot{\theta}=p^{2} \sin (\alpha+\theta)+\frac{m \ddot{x}_{g}}{I_{0}} R \cos (\alpha+\theta)+\frac{m \ddot{y}_{g}}{I_{0}} R \sin (\alpha+\theta) \text { for } \theta<0
\end{gathered}
$$

where $p^{2}=m g R / I_{0}$.

As defined in (Chatzis et al., 2016), in pattern C, static equilibrium is achieved by the reaction forces $f_{x}$ and $f_{y}$ as shown in Figure 1 , the latter acting at a distance $d$ from 0 . Due to the assumption of non tensile stresses provided by the contact interface, these forces must lie within the base and rocking will initiate when the vertical reaction reaches a corner, i.e. when $d \rightarrow 0$ or $d \rightarrow 2 b$.

As the block rocks, transition from pattern A to B involves an impact between the block and the ground as shown in the following Figure 2.

[FIG. 2 about here.]

The distributed impulses acting along the base of the block during impact can be represented by horizontal and vertical resultants $J_{x}$ and $J_{y}$. The location of the vertical resultant is defined as being at a distance $\lambda b$ from the post-impact 
rocking corner. In (Chatzis et al., 2016), it was observed that there is an infinite number of admissible distributions of vertical impulses along the base during the impact, and as a result the parameter $\lambda$ possesses an inherent uncertainty. Using impulse-momentum equations, the post-impact angular velocity can be expressed in terms of the pre-impact velocity $\dot{\theta}^{-}$and $\lambda$. This relation is most conveniently expressed by the reduction of energy factor $r$, which is the ratio of the kinetic energy after and before the impact:

$$
r=\frac{\left(\dot{\theta}^{+}\right)^{2}}{\left(\dot{\theta}^{-}\right)^{2}}=\left(\frac{1-\frac{3}{4}(2-\lambda) \sin ^{2} \alpha}{1-\frac{3}{4} \lambda \sin ^{2} \alpha}\right)^{2}
$$

The observation that the system's energy cannot increase during the impact, together with the assumption that the ground cannot exert tensile forces, requires $\lambda$ to take a value between 0 and 1 , that is, the resultant may act anywhere between the post-impact rocking corner and the midpoint of the base. Finally, it should be noted that $\sqrt{r}=\frac{1-\frac{3}{4}(2-\lambda) \sin ^{2} \alpha}{1-\frac{3}{4} \lambda \sin ^{2} \alpha}$ should be positive. Negative solutions do not make physical sense and would result in a free-flight pattern that is incompatible with the IPM.

In the original analysis by (Housner, 1963), and in most subsequent studies, the vertical impulse during the impact was assumed to act at the post-rocking corner, meaning that $\lambda$ was taken as zero. However, the true value of $\lambda$ depends on several factors including the unmodeled properties of the contact interface. In (Chatzis et al., 2016), it was shown that the $\lambda=0$ assumption is non-conservative, with higher values of $\lambda$ resulting in increased probability of toppling after impact. This is to be expected, as equation 2 shows that larger values of $\lambda$ correspond to a smaller energy loss during impact, with no energy loss when $\lambda=1$.

\section{Dynamic model: Rocking patterns for two stacked blocks}

This section extends the IPM to the case of two stacked rigid blocks, symmetric about the vertical axis. The geometry and notation for this case is shown in Figure 3(a), and is consistent with that used by (Spanos et al., 2001). The blocks each have a mass $m_{i}$, where $i=1$ corresponds to the bottom body and $i=2$ to the upper. The moment of inertia of body $i$ about its centre of mass, $G_{i}$, is denoted as $I_{G_{i}}$. Their half-height and half-width are defined respectively as $h_{i}$ and $b_{i}$. The right bottom corners of the blocks are denoted as $O_{i}$ and the left $O_{i}^{\prime}$. The distance between the centres of mass and $O_{i}$ is defined as $R_{i}$ and the angle between $R_{i}$ and the side of the corresponding block as $\alpha_{i}$. The distances between corners $O_{1}$ and $O_{2}$ is $l$ and the angle between $l$ and the side of the block is $\beta$. The distance between $O_{1}$ and $O_{2}^{\prime}$ is $l^{\prime}$ and the angle between $l^{\prime}$ and the side of the block is $\beta^{\prime}$. The rotation of each body is defined to be positive clockwise and denoted as $\theta_{1}$ and $\theta_{2}$ for the bottom and top body respectively. When the bodies are fully connected, the moment of inertia of the system about its centre of mass, $G$, is represented as $I_{G}$, the distance between the centre of mass and $O_{i}$ 
is defined as $R$ and the angle between $R$ and the side as $\alpha$.

[FIG. 3 about here.]

There are eight possible dynamic rocking patterns for the stacked rocking bodies, aside from the static configuration where both bodies are at rest corresponding to the case shown in Figure 3(a). There are four basic categories of dynamic patterns, as illustrated in Figure 4, indicated by a numerical index, each with two symmetrical sub-patterns that are indicated by the letters $a$ and $b$. Depending on the sign of $\theta_{1}$ and $\theta_{2}-\theta_{1}$ respectively, the bottom and top bodies rock with respect to one of their bottom corners. The cases for which $\theta_{1}$ or $\theta_{2}-\theta_{1}$ become equal to zero result in the bottom or top contact interface respectively being the line segment between the two bottom corners of the bottom and top body respectively. This situation will be referred to from hereafter by denoting the corresponding interface as 'active'. Following the assumptions of the IPM, each body can then be simulated as a pendulum whose pin is located at that corner. Therefore, as in the case of a single body, this implies that the bodies cannot slide or experience free-flight, that the ground and bodies are rigid and that the response of the system is strictly planar.

[FIG. 4 about here.]

\section{Kinematics and equations of motion}

Based on the previous assumptions any pattern can be described using at most two generalised coordinates, $\theta_{1}$ and $\theta_{2}$. The equations of motion for any pattern can then be derived using Lagrange's method (Greenwood, 2003). In its generalised form this is given by:

$$
\frac{d}{d t}\left(\frac{\partial T}{\partial \dot{\theta}_{i}}\right)-\frac{\partial T}{\partial \theta_{i}}=Q_{i} \quad Q_{i}=\sum_{j=1}^{n} F_{j} \frac{\partial r_{j}}{\partial \theta_{i}}
$$

where $T$ is the kinetic energy of the system, $Q_{i}$ is the generalised force, $n$ is the number of applied forces, $F_{j}$ is an external force vector applied on a point at a vectorial distance $r_{j}$ from $O_{1}$ as shown in Figure 3(b). The forces that are applied here correspond to the gravitational forces applied to the two bodies $m_{i} g$ and the inertial forces due to a ground accelerating horizontally $\ddot{x}_{g}$. The kinetic energy of the system is given by:

$$
T=\frac{1}{2} \sum_{i=1}^{2}\left(m_{i} v_{i}^{2}+I_{G i} \dot{\theta}_{i}^{2}\right) \quad v_{i}^{2}=\dot{x}_{i}^{2}+\dot{y}_{i}^{2}
$$

where $v_{i}$ is the relative velocity to the ground of the centre of mass of the block $i$. Hence, the translational kinetic energy appearing in equation (4) is not equal 
to the total translational energy of the body. This is due to the fact that the ground accelerations are used in equation (4) as inertia forces (Chatzis and Smyth, 2012a).

Using the coordinate system defined in Figure 3(b), the positions of the centres of mass of the blocks $G_{1}$ and $G_{2}$ are given, for patterns 1a, 1b, 2a, 2b, 4a and 4b, by the following equations:

$$
\begin{aligned}
& x_{1}=H_{\theta_{i}} 2 b_{1}+S_{\theta_{1}} R_{1} \sin \left(\alpha_{1}-S_{\theta_{1}} \theta_{1}\right) \quad y_{1}=R_{1} \cos \left(\alpha_{1}-S_{\theta_{1}} \theta_{1}\right) \\
& \begin{aligned}
x_{2}= & H_{\theta_{i}} 2 b_{1}+S_{\theta_{1}} S_{l} \sin \left(\theta_{1}-S_{\theta_{1}} S_{\beta}\right) \\
& +S_{\theta_{2}} R_{2} \sin \left(\alpha_{2}-S_{\theta_{2}} \theta_{2}\right)
\end{aligned} y_{2}=S_{l} \cos \left(\theta_{1}-S_{\theta_{1}} S_{\beta}\right)+R_{2} \cos \left(\alpha_{2}-S_{\theta_{2}} \theta_{2}\right)
\end{aligned}
$$

The signum function $S_{\theta_{i}}, S_{\beta}, H_{\theta_{i}}$ and $S_{l}$ are defined as:

$$
\begin{gathered}
S_{\theta_{i}}=\left\{\begin{array}{ll}
1 & \text { for } \theta_{i} \geq 0, \\
-1 & \text { for } \theta_{i}<0
\end{array} \quad S_{\beta}= \begin{cases}\beta & \text { for } \theta_{1}>0, \theta_{2}>0 \text { or } \theta_{1}<0, \theta_{2}<0, \\
\beta^{\prime} & \text { for } \theta_{1}>0, \theta_{2}<0 \text { or } \theta_{1}<0, \theta_{2}>0,\end{cases} \right. \\
H_{\theta_{i}}=\left\{\begin{array}{ll}
0 & \text { for } \theta_{i} \geq 0, \\
1 & \text { for } \theta_{i}<0
\end{array} \quad S_{l}= \begin{cases}l & \text { for } \theta_{1}>0, \theta_{2}>0 \text { or } \theta_{1}<0, \theta_{2}<0, \\
l^{\prime} & \text { for } \theta_{1}>0, \theta_{2}<0 \text { or } \theta_{1}<0, \theta_{2}>0,\end{cases} \right.
\end{gathered}
$$

For the case of pattern 3 , as $\theta_{2}=\theta_{1}$ the equivalent centre of mass of the system, $G$, is shown in Figure 3(a) and its position is defined as:

$$
x_{G}=H_{\theta_{i}} 2 b_{1}+S_{\theta_{1}} R \sin \left(\alpha-S_{\theta_{1}} \theta_{1}\right) \quad y_{G}=R \cos \left(\alpha-S_{\theta_{1}} \theta_{1}\right)
$$

where $R=\sqrt{h^{2}+b_{1}^{2}}, h=\frac{m_{1} h_{1}+m_{2}\left(2 h_{1}+h_{2}\right)}{M}, M=m_{1}+m_{2}$ and $I_{G}=I_{G_{1}}+I_{G_{2}}+$ $m_{1}\left(h-h_{1}\right)^{2}+m_{2}\left(h-h_{2}\right)^{2}$. To use equation (3) for this case only one generalised coordinate, e.g. $\theta_{1}$ is used after replacing $\theta_{2}=\theta_{1}$, before calculating the energy of the system $T=\frac{1}{2}\left(M v_{G}^{2}+I_{G i} \dot{\theta}_{i}^{2}\right)$. Similarly for pattern 4 one uses only $\theta_{2}$ as a generalised coordinate and takes into account that $\theta_{1}=0$ before using equation (4). The equation of motions for all the possible cases are presented in (Spanos et al., 2001), and are omitted in this paper for reasons of brevity.

The rocking motion of the system involves transitions from one pattern to another. Those transitions may occur with or without an impact and are described in the following sections.

\section{Change of pattern without impact}

In this category, a change of patterns occurs without an impact. In fact, as will be described in this section the transitions occur due to the inabilities of the contact interfaces to provide tensile stresses. This is of course an extension of the 
assumption that was used in the IPM and allows for the transition between the static and dynamic patterns as described in (Chatzis et al., 2016).

It is useful to cluster different patterns depending on whether the contact interface between the bottom body and the ground and the one between the two bodies, which will be referred to as bottom and top interface respectively, correspond to a point or a linear segment. The two contact interfaces will be referred to as inactive or active respectively. Taking into account the symmetry between patterns $a$ and $b$ the following three cases presented in Figure 5 are of interest.

[FIG. 5 about here.]

In the case of Figure 5(a), which is the static pattern, both contact interfaces are active. Figure 5(b) corresponds to the case where the bottom interface is active and the top is inactive and while the figure shows pattern $4 a$, the case is applicable to $4 b$ as well. The last case is when the bottom interface is inactive and the top is active, Figure 5(c). Similarly, while Figure 5(c) corresponds directly to pattern $3 a$ the discussion is applicable to $3 b$ as well.

Figure 5 further shows the tangential and normal interface forces, $f_{x_{i}}$ and $f_{y_{i}}$, occuring at interface $i$. For clarity, only the reaction forces applied to the body above the interface are drawn. It should be noted that when an interface is inactive its reactions have to pass through the corresponding rocking corner. For an active interface however, the reaction forces could act at any point between the two corresponding corners. The distances along the interface from the right towards the left corner are denoted in Figure 5 as $d_{i}$.

Furthermore, $d_{i} / b_{i} \in[0,2]$, i.e., the vertical reaction forces of an active interface have to be applied between the two corners. Otherwise the non-tensile forces assumption for the interfaces would be violated. This now enables us to define the condition for transition between patterns without impact: A pattern will be sustained when the reaction forces of each of the active interfaces remain between the corresponding corners. At the instant at which the reaction forces of an active interface are applied at one of the corners, i.e., $d_{i} \rightarrow 0$ or $d_{i} / b_{i} \rightarrow 2$ then a transition occurs to a corresponding pattern where the active interface of interest has been replaced with an inactive interface involving that corner. Hence, the transitions that are expected to occur and the corresponding conditions can be summarised in Table 1.

[TABLE 1 about here.]

The parameters $d_{i}, f_{x_{i}}$ and $f_{y_{i}}$ can easily be obtained through Newton's equations of motion for the two bodies:

$$
m_{i} \ddot{x}_{i}=\sum_{j} \mathbb{F}_{x_{i}}^{j} \quad m_{i} \ddot{y}_{i}=\sum_{j} \mathbb{F}_{y_{i}}^{j} \quad I_{G_{i}} \ddot{\theta}_{i}=\sum_{j} \mathbb{M}_{G_{i}}^{j}
$$


where $\mathbb{F}_{x_{i}}^{j}, \mathbb{F}_{y_{i}}^{j}$ correspond to the force $j$ applied to body $i$ along the directions $x$ and $y$ respectively, and $\mathbb{M}_{G_{i}}^{j}$ correspond to the moment $j$ applied to body $i$ with respect to its centre of mass $G_{i}$. Solving the system of those equations is straightforward as the accelerations $\ddot{\theta}_{i}$ can be calculated first using, the equations of motion (3).

This section has described how transitions between patterns without impact can be described by tracking the values of $d_{i}$. The resulting transitions are equivalent to those defined in (Spanos et al., 2001). However, the approach presented here allows for easier implementation in algorithms that use Event detection (as discussed later) and allows a better understanding of the mechanics that lead to transitions.

\section{Transitions between patterns with impact}

In this category the change of patterns follows the event of an impact. An impact occurs when either $\theta_{1}$ or $\theta_{2}-\theta_{1}$ change sign. The contact surfaces are considered to be rigid following the assumptions of the IPM, hence the duration of the impact is considered to be infinitesimally small. It is important to distinguish between three time instants: just before impact, denoted by the superscript ${ }^{+}$, just after impact, associated denoted by the superscript ${ }^{-}$, and a time instant during the infinitesimally small duration of the impact, referred to as the time instant of the impact. The pre- and post- impact instants are associated with one of the patterns in Figure 4. Table 2 shows the possible transitions; $P 1$ and $P 2$ refer to the points of application of the vertical impulses for the lower and upper interface, respectively. The arrow points at the distance of $P_{i}$ from the corresponding future rocking corner of that interface.

[TABLE 2 about here.]

It is important to note that during the impact the system does not belong to any pattern. Following the convention of the previous section, the top or bottom contact interface is referred to as active or inactive during the impact depending on whether the contact interface corresponds to a line segment or a corner respectively. During an impact at least one of the two interfaces has to be active. Figure 6 describes three distinct cases that occur during impact.

[FIG. 6 about here.]

During impact, impulsive forces are applied at both bodies from the contact interfaces. The corresponding tangential and normal impulses from contact interface $i$ are denoted as $J_{x_{i}}$ and $J_{y_{i}}$ respectively. For reasons of clarity only the impulses acting from the contact interface to the body above are drawn in Figure 6. It is important to note that for an inactive contact, as there is a single point of contact, the corresponding impulses have to pass through that point. For an active contact though, the resultant normal impulses may be applied at any point 
along the contact interface. This is accounted for using a corresponding tangential distance from the future rocking corner denoted as $\lambda_{i} b_{i}$, where $i=1$ and 2 correspond to the bottom and top interface respectively. The following Figure 7 associates the resultant normal impulse $J_{y_{i}}$ of contact interface $i$ to the impulse distribution it replaces.

[FIG. 7 about here.]

The assumption of non-tensile stresses used by the model in section Inverted Pendulum model for a single block, which allows for the change of patterns without impact further leads to the lack of non-tensile impulses in the contact interfaces during an impact. As can be seen in Figure 7, and equivalently to what was shown for single rocking bodies in (Chatzis et al., 2016), the location of the resultant normal impulses has to be between the two corners or otherwise the assumption of non-tensile stresses would be violated. As a result: $\lambda_{i} / b_{i} \in[0,2]$.

An important difference with the work of (Spanos et al., 2001) is that the assumption that $\lambda_{i}=0$, i.e., that angular momentum about the future rocking corner is conserved during impact, is not made. All values of $\lambda_{i}$ between 0 and 2 are compatible with the assumptions underlying the model. However, for a given type of impact, block dimensions and pre-impact velocities, some values of $\lambda_{i}$ may result in an increase in the energy of the system during impact. Since this condition is physically impossible, such values of $\lambda_{i}$ must be excluded. For single rocking blocks this restriction requires $\lambda$ to be less than 1 , but this is not necessarily the case for stacked blocks. Nevertheless, in the following, the focus is limited to values of $0 \leq \lambda_{i} \leq 1$, while ensuring that the chosen values of $\lambda_{i}$ do not lead to energy increase during any impact.

Having defined the location of the resultant contact impulses for each interface and for any transition, it should be highlighted that for body 2 the only external, non-zero, impulses applied at the body during the impact are $J_{x_{2}}$ and $J_{y_{2}}$. Similarly for the system of the two rocking bodies, the impulses externally applied to the system are $J_{x_{1}}$ and $J_{y_{1}}$. As a result, during any impact, the angular momentum of the upper body will be conserved with respect to the point of application of $J_{y_{2}}, P_{2}$, and the angular momentum of the whole system will be conserved with respect to the point of application of $J_{y_{1}}, P_{1}$. This is expressed in the following two equations.

$$
H_{2 \rightarrow P_{2}}^{+}=H_{2 \rightarrow P_{2}}^{-} \quad \sum_{i=1}^{2} H_{i \rightarrow P_{1}}^{+}=\sum_{i=1}^{2} H_{i \rightarrow P_{1}}^{-}
$$

where $H_{i \rightarrow P_{j}}^{ \pm}$corresponds to the angular momentum of body $i$ with respect to point $P_{j}$ defined as:

$$
H_{i \rightarrow P_{j}}^{ \pm}=I_{G_{i}} \dot{\theta}_{i}^{ \pm}+m_{i}\left(\mathbf{r}_{P_{j} \rightarrow G_{i}} \times \mathbf{v}_{G_{i}}^{ \pm}\right) \cdot \hat{\mathbf{k}}
$$


This is now illustrated using Figure 8, where the system switches form pattern $3 \mathrm{a}$ to $1 \mathrm{~b}$. Angular momentum of the whole system is conserved about the impact point, at a distance $\lambda_{1} b_{1}$ form $O_{1}^{\prime}$. Leading to the following equation:

$$
A_{1} \dot{\theta}_{1}^{-}+B_{1} \dot{\theta}_{2}^{-}=C_{1} \dot{\theta}_{1}^{+}+D_{1} \dot{\theta}_{2}^{+}
$$

where,

$$
\begin{aligned}
A_{1}=I_{O}-\left(2-\lambda_{1}\right) M b_{1}^{2} \quad C_{1} & =I_{O 1}-\lambda_{1} m_{1} b_{1}^{2}+m_{2} l\left(2 h_{1}+h_{2}\right) \cos \beta \\
& +\left(1-\lambda_{1}\right) m_{2} l b_{1} \sin \beta \\
B_{1}=0 & D_{1}=I_{G 2}+m_{2} R_{2}\left(2 h_{1}+h_{2}\right) \cos \alpha_{2} \\
& +\left(1-\lambda_{1}\right) m_{2} R_{2} b_{1} \sin \alpha_{2}
\end{aligned}
$$

where $M=m_{1}+m_{2}, I_{O}=I_{O 1}+I_{G 2}+m_{2} R_{2 O}^{2}$ and $I_{O_{i}}$ are the moments of inertia of the blocks about their corners. Note that in this case both blocks behave as one, i.e., $\dot{\theta}_{2}^{-}=\dot{\theta}_{1}^{-}$. A second equation can be derived by considering the conservation of angular momentum about $P_{2}$ for block 2 alone leading to:

$$
A_{2} \dot{\theta}_{1}^{-}+B_{2} \dot{\theta}_{2}^{-}=C_{2} \dot{\theta}_{1}^{+}+D_{2} \dot{\theta}_{2}^{+}
$$

where,

$$
\begin{array}{rlrl}
A_{2} & =I_{O_{2}}-\lambda_{2} m_{2} b_{2}^{2}+m_{2} l^{\prime} h_{2} \cos \left(\beta^{\prime}\right) & & B_{2}=0 \\
& -\left(1-\lambda_{2}\right) m_{2} l^{\prime} b_{2} \sin \left(\beta^{\prime}\right) & & \\
C_{2} & =m_{2} l h_{2} \cos \beta+ & D_{2}=I_{O 2}-\lambda_{2} m_{2} b_{2}^{2}
\end{array}
$$

The post impact velocities of the stacked bodies can be calculated solving equations (13) and (14). Note that the solutions depend on the assumed values of $\lambda_{1}$ and $\lambda_{2}$.

[FIG. 8 about here.]

The same procedure can be followed for all the transitions with impacts and the results can always be presented in the form of equations (13) and (14), with different expressions for the coefficients $A_{1}, A_{2}, B_{1}, B_{2}, C_{1}, C_{2}$ and $D_{1}, D_{2}$. Equations for the rest of the patterns transitions described in Table 1 are presented in Appendix A. The coefficients are functions of the dimensions of the two bodies and also potentially the rotations of the bodies and finally are affected for any case by at least one of $\lambda_{1}$ and $\lambda_{2}$. If one sets $\lambda_{1}=0$ and $\lambda_{2}=0$ then the same expressions are obtained as those presented in (Spanos et al., 2001). It is evident from equations (13) and (14), that the post impact rotational velocities and hence the future trajectory of the body depend on the values of the assumed $\lambda_{i}$. 
In this case, it should be noted that there is a design choice that can affect the values of $\lambda_{i}$ at the two interfaces. This is either the introduction of an absorbing material between the two bodies around that interface, e.g., sorbothane rubber as in (Smyth et al., 2016), or the use of physical feet at the corners as explained in (Chatzis and Smyth, 2012c; Chatzis et al., 2016). Assuming that the introduction of the rubber material does not substantially change the stiffness of the contact interface, its effect in the model examined here is an increased exchange of energy between the two bodies of that interface (Chatzis et al., 2016). This in essence corresponds to decreasing the corresponding value of $\lambda_{i}$ at that interface. Hence, placement of the rubber material at the bottom or top interface decreases the value of $\lambda_{1}$ and $\lambda_{2}$ respectively towards zero in comparison to the value of those parameters for the original interface. Equally the works presented in (Chatzis and Smyth, 2012c; Chatzis et al., 2016) describe of how placement of physical feet would lead to decreasing the value of $\lambda_{i}$. This second option is mainly useful for the bottom interface.

\section{Special transition cases}

Consider again the case when the blocks are in pattern $3 \mathrm{a}$ and a transition with impact leading to pattern $1 \mathrm{~b}$ occurs as shown in Figure 8. If after the impact $\dot{\theta}_{2}^{+}-\dot{\theta}_{1}^{+}<0$, the transition to pattern $1 \mathrm{~b}$ is possible. However, if the analysis results in $\dot{\theta}_{2}^{+}-\dot{\theta}_{1}^{+}>0$, this would result in a subsequent immediate impact activating the upper interface. These successive impacts with no progress of time are not physically possible and even mathematically would lead to erroneous results. That is because pattern $1 \mathrm{~b}$ is not well defined for $\theta_{1}=\theta_{2}$ and $\dot{\theta}_{2}-\dot{\theta}_{1}>0$. This situation may occur for several transitions with impact for which the sign of either $\dot{\theta}_{1}^{+}$or $\dot{\theta}_{2}-\dot{\theta}_{1}$, together with the post impact values of $\theta_{1}$ and $\theta_{2}$ are not compatible with the post impact pattern. Table 3 presents the necessary conditions on the post impact angular velocities for the different transitions with impact to be feasible.

[TABLE 3 about here.]

If the conditions are violated then the investigated transition is not feasible. This case is also investigated in (Spanos et al., 2001) and the treatment suggested is to instead switch to a post impact pattern for which the function that violated the sign condition, either $\dot{\theta}_{1}$ or $\dot{\theta}_{2}$, is zero. This results in changing the post impact pattern as also described in Table 3.

However, in order for the new post-impact patterns to be feasible either or both of the conditions, $\dot{\theta}_{1}=0$ or $\dot{\theta}_{2}-\dot{\theta}_{1}=0$, have to be satisfied. Those conditions act in parallel with the momentum equations (12). As a result the values of $\lambda_{i}$ cannot be chosen freely any more. Within the context of the work presented in (Spanos et al., 2001) this means that during such instances the assumption of conserving angular momentum with respect to a future rocking corner, i.e. $\lambda_{i}=0$, cannot be satisfied for the active contact interface. This, of course, is not violating any of the assumptions of the model as described in this paper, it is 
however a further indication that even in the work of (Spanos et al., 2001) values of $\lambda_{i}$ other than zero are used for such cases. Within the context of the current work, such instances would result in changing the corresponding $\lambda_{i}$ to the specific value that guarantees the transition. Table 3 shows the points of application of the vertical impulses for both interface.

Going back to the example presented, to avoid the non-feasible post impact result $\dot{\theta}_{2}^{+}-\dot{\theta}_{1}^{+}>0$, it is assumed that the system switches to pattern $3 b, \dot{\theta}_{2}^{+}=\dot{\theta}_{1}^{+}$. In this case the analysis reduces to a single rocking body analysis (Chatzis et al., 2016) and conservation of angular momentum of the whole system about the bottom impact force location yields:

$$
\dot{\theta}_{1}^{+}=\frac{I_{0}-\left(2-\lambda_{1}\right) M b_{1}^{2}}{I_{0}-\lambda_{1} M b_{1}^{2}} \dot{\theta}_{1}^{-}
$$

Equivalently one can obtain the same expression more generally by using the conservation of angular momentum equation for the system with respect to $P_{1}$ and replacing $\dot{\theta}_{2}^{+}=\dot{\theta}_{1}^{+}$for any value of $\lambda_{1}$. However, the conservation of angular momentum of the upper body together with the condition $\dot{\theta}_{2}^{+}=\dot{\theta}_{1}^{+}$, uniquely prescribe the value of $\lambda_{2}$ :

$$
\begin{array}{ccc}
\lambda_{2}=\frac{A_{3} \dot{\theta}_{1}^{-}+B_{3} \dot{\theta}_{1}^{+}}{C_{3} \dot{\theta}_{1}^{-}+D_{3} \dot{\theta}_{1}^{+}} & \\
& \\
A_{3}=-\left(I_{O_{2}}+m_{2} l^{\prime} h_{2} \cos \beta^{\prime}\right. & B_{3}=I_{O_{2}}+m_{2} l h_{2} \cos \beta \\
\left.-m_{2} l^{\prime} b_{2} \sin \beta^{\prime}\right) & +m_{2} l b_{2} \sin \beta \\
C_{3}=m_{2} b_{2}\left(l^{\prime} \sin \beta-b_{2}\right) & D_{3}=m_{2} b_{2}\left(l \sin \beta+b_{2}\right)
\end{array}
$$

Note that, in equation (18) $\dot{\theta}_{1}^{+}$is also a function of $\lambda_{1}$. This measn that $\lambda_{2}$ has to have a very specific value given the value attained by $\lambda_{1}$, the dimensions of the body and the pre-impact velocities.

Similarly, it is possible that following the previously studied switch between patterns $3 a \rightarrow 1 b$, where after solving the momentum equations $(12), \dot{\theta}_{1}^{+}$is found to be positive. As discussed earlier the solution suggested to tackle the occurring problem is through setting $\dot{\theta}_{1}^{+}=0$ and choosing pattern $4 \mathrm{~b}$. This can be seen in Figure 9.

[FIG. 9 about here.]

For the above transition, the angular moment equations for the system (13) and the upper block (14) take the form:

$$
A_{1} \dot{\theta}_{1}^{-}=D_{1} \dot{\theta}_{2}^{+} \quad A_{2} \dot{\theta}_{1}^{-}=D_{2} \dot{\theta}_{2}^{+}
$$

where $A_{1}, A_{2}, D_{1}$ and $D_{2}$ are previously defined. 
In this transition both interfaces are active and hence, only $\lambda_{i}$ are unknown. The locations of both contac points $P_{1}$ and $P_{2}$ are functions of the value of $\lambda_{1}$ and $\lambda_{2}$, respectively. There are now two momentum equations and three unknowns $\dot{\theta}_{2}^{+}, \lambda_{1}$ and $\lambda_{2}$. If one of the $\lambda_{i}$ is specified the other is uniquely defined as the number of unknowns decreases to two. For example, if $\lambda_{2}$ is assumed, equations in (17) can be solved for $\dot{\theta}_{2}^{+}=\frac{A_{2}}{D_{2}} \dot{\theta}_{1}^{-}$and $\lambda_{1}$ :

$$
\lambda_{1}=\frac{A_{3} \dot{\theta}_{1}^{-}+B_{3} \dot{\theta}_{2}^{+}}{C_{3} \dot{\theta}_{1}^{-}+D_{3} \dot{\theta}_{2}^{+}}
$$

$$
\begin{array}{rlrl}
A_{3}=I_{O}-2 M b_{1}^{2} & B_{3} & =-\left(I_{G 2}+m_{2} R_{2}\left(2 h_{1}+h_{2}\right) \cos \alpha_{2}\right. \\
& \left.+m_{2} R_{2} b_{1} \sin \alpha_{2}\right) \\
C_{3}=-\left(M b_{1}^{2}\right) & D_{3}=m_{2} R_{2} b_{1} \sin \alpha_{2}
\end{array}
$$

Similar computations apply to all transitions in this category. However, while this formulation alleviates the mathematical problems of the model, from a physical point of view it is questionable that the impulses would be applied exactly at the locations that satisfy the additional constraints. Instead, it is more probable that when such situations are encountered some of the remaining assumptions on the post impact behaviour would be violated. It has been noticed in the literature that for the case of a single rocking body, the occurrence of such an instance, i.e., when the coefficient of restitution becomes negative, indicates that in reality the body would bounce during impact (Shenton and Jones, 1991; Lipscombe and Pellegrino, 1991). It is hence expected that equally in the case of stacked rocking bodies, the bodies around at least one of the contact interfaces are expected to become separated after the impact when the situation in Table 2. As a result, part of the system is expected to violate the assumption of no bouncing and hence proper study of the system in such scenarios would require the use of a model that takes free-flight and sliding modes into account, such as the extension of the models (Shenton and Jones, 1991; Chatzis and Smyth, 2012c).

Hence, for the remainder of this paper it will be ensured that this problem does not occur for any of the systems and excitations studied.

\section{Numerical Formulation}

Following the numerical simulation of (Chatzis et al., 2016) for the single body case, the equations of motion for each pattern are brought to state-space form and integrated over time numerically. A variable time-stepping Runge-Kutta 4-5 is applied (Dormand and Prince, 1980) which uses a pair of a fourth-order verified against a fifth order Runge-Kutta to propagate the solution over time.

The equations describing the stacked bodies problem are not smooth and the angular velocities $\dot{\theta}_{i}$ are discontinuous around the time instant of impact. To switch between patterns, the event-driven function algorithm suggested by (Shampine and Gordon, 1975) is used. The algorithm allows stopping the integration around a specific time at which an event occurs. The events are the 
transitions between patterns or the toppling of the blocks. Those are located online by the algorithm as implemented in Matlab's ode45 solver with the Events option (MathWorks 2016) (Shampine and Gordon, 1975).

Figure 10 shows a flowchart of the design structure of the program. The 'simulation parameters' comprise of the geometrical parameters of the blocks, the initial conditions of the blocks and the base accelerations. 'Static state' is the case where $\theta_{1}=\theta_{2}=\dot{\theta}_{1}=\dot{\theta}_{2}=0$. The condition to initiate the motion is determined at $d_{i} / b_{i} \rightarrow 0$ or/and $d_{i} / b_{i} \rightarrow 2$ depending on the pattern. Here, ' $a(t)$ finished' is used to denote that the duration of the input is over. $t_{\text {end }}$ is the predetermined simulation time, usually defined as the overall duration of excitation. The "static pattern' is a dummy ode which simply outputs the conditions of the steady state until one of the conditions for initiation of rocking is satisfied.

[FIG. 10 about here.]

\section{STABILITY DIAGRAMS}

Initially the stability of the system will be investigated for ground accelerations having the form of an horizontal sinusoidal pulse as in (Zhang and Makris, 2001; Chatzis et al., 2016). This input, despite its simplicity, allows for demonstrating important features of the system (Zhang and Makris, 2001; Plaut et al., 1996). The blocks are subjected to single- and double-cycle sinusoids defined as:

$$
\ddot{x}_{g}=0, \quad \text { if } t>N_{c} T \quad \ddot{x}_{g}=u_{0} \sin \left(\omega_{u} t\right), \quad \text { if } 0 \leq t \leq N_{c} T
$$

where $u_{0}$ is the amplitude and $\omega_{u}$ the frequency of the pulse, with a period of $T=2 \pi / \omega_{u}$ and $N_{c}$ corresponds to the number of cycles.

In the following, a stability diagram is obtained by varying the values of $u_{0}$ and $\omega_{u}$ and recording for each case of whether any of the two bodies topple or the system survives the excitation. The toppling of a body will be referred to in the following as failure of the system.

For the system examined here, the outcomes can be classified in terms of areas of $u_{0}$ and $\omega_{u}$ associated with: survival, failure with no impacts occurring, failure with impacts where only one of the two interfaces is active, or where both of them are active. Note that for all the areas for which an impact occurs, the values of $\lambda_{i}$ related to the active interfaces during any of the impacts have an effect on the outcome. Finally, it may be of interest for some system to further distinguish between failure of only the upper body which occurs when $\left|\theta_{1}-\theta_{2}\right|=\pi / 2$ and failure of the bottom body occurring when $\theta_{1}=\pi / 2$. The latter type of failure is certainly associated with failure of the system, as it is inevitable that past this point the upper body will also topple.

\section{Change of pattern}

In this section, a system of stacked bodies is subjected to single cycle sinusoidal acceleration pulses. Initially the investigated system involves two blocks that have 
the same aspect ratio $h_{i} / b_{i}=3$ and dimensions: $h_{1}=60 \mathrm{~cm}, b_{1}=20 \mathrm{~cm}, h_{2}=15$ $\mathrm{cm}, b_{2}=5 \mathrm{~cm}$. It will initially be assumed that $\lambda_{1}=\lambda_{2}=0$, i.e., the impulses are located at the future rocking corners. The stability diagram of the system is presented in Figure 11 where the horizontal and vertical axes are normalised with $p=6.8215$ and $\alpha=0.3218$ corresponding to the properties of block 2 .

[FIG. 11 about here.]

Figure 11(a) presents the stability diagram separating the failure regions depending on whether there were any impacts prior to failure and if so further specifies which interfaces were active during any of those impacts. Figure 11(b) presents the stability diagram separating the failure regions depending on which body toppled first. The non-linearity of the problem is apparent in Figure 11(a) as for example for pulses of constant frequency, as the amplitude $u_{0} /(\alpha g)$ is increased the system may transition from survival, to failure with impact with both interfaces active, back to survival, followed by failure with only the bottom interface active, then re-transition to failure with both interfaces active and finally to failure where only the top interface is active.

In Figure 11, as in the case of a single rocking body, for low amplitudes the body remains in the static pattern without ever transitioning to a dynamic pattern. For the system examined here the body always transitions first to pattern $3 \mathrm{a}$ and hence the amplitude that separates the static pattern from the dynamic patterns is $u_{0} /\left(\tan \left(b_{1} / h\right) g\right)<1$. An additional region that that resembles a behaviour encountered in the single body stability diagram is the region of points where failure occurs without impact. As an impact does not occur in that region the outcome is not affected by the assumed value of $\lambda_{i} / b_{i}$. Unlike the single rocking body case where the region of failure without impact has a lower boundary in terms of the amplitudes but not an upper boundary, in this problem the region can be separated into an upper lobe where indeed increase of $u_{0}$ results in continuously remaining in this region, and a lower lobe where eventually as $u_{0}$ is increased an impact is experienced. Equally, if failure occurs with an interface $i$ never becoming active, then the associated assumption on the value of $\lambda_{i}$ does not influence the survival or failure of the body for that combination of $u_{0}$ and $\omega_{u}$ values.

In Figure 11(b) the failure regions are presented in terms of which is the first body that experiences toppling. It can be seen that the majority of the regions of failure are associated with toppling of the top body. In fact the region of failure for body 1 (and hence eventually the system) is a region of high amplitudes and low frequencies surrounded by a region of where the top body fails first. A careful comparison between the outer boundaries of the area of failure for body 1 in Figure 11(b) versus the region of failure without impact in Figure 11(a) shows that the former matches the upper lobe of the latter.

To understand this behaviour, consider the following two single-body rocking systems: when the bottom body is fixed but the upper body rocks, and when 
the two bodies always remain fully connected with the top interface active and this overall system rocks as one body. The former case will be referred to as case $\mathrm{B}$ and is associated with patterns $4 a$ and $4 b$, while the latter will be referred to as case $\mathrm{C}$ and is associated with patterns $3 a$ and $3 b$. The original problem of both bodies rocking will be referred to as case A. Figure 12 shows the stability diagrams for cases B and C while Figure 13 draws comparisons with the stability of the system of stacked rocking bodies shown in Figure 11.

[FIG. 12 about here.]

[FIG. 13 about here.]

It can be seen in Figure 13 that, for the specific system studied and the assumption of $\lambda_{1}$ and $\lambda_{2}$ being zero, case $\mathrm{C}$ appears to be overall more stable. Case $\mathrm{B}$ is in general more stable than $\mathrm{A}$, although there are areas in the diagram where the latter would survive but the former will not. This investigation presented in Figure 13 is useful in terms of design as for example the dimensions studied here could be encountered in the case of an artifact (body 2) situated on top of a pedestal (body 1). Then the design question would be of whether both bodies should be allowed to rock, the pedestal should be clamped on the floor, or the artifact should be clamped on the pedestal which would be allowed to rock. The three design scenarios correspond to cases $\mathrm{A}, \mathrm{B}$ and $\mathrm{C}$ respectively. It appears that for the case studied here the scenario of clamping the artifact to the pedestal would result overall in less toppling of the system.

The upper lobe of the area of failure with no impact, where block 1 fails first, presented in Figure 11(a) can now be revisited. As the system experiences no impacts in this region of the $a_{o}$ and $\omega_{u}$ map, the dynamics of the body are dictated by only two dynamic patterns: initially $3 b$ (or $3 a$ ) and potentially $1 b$ (or $1 a$ ). In case $\mathrm{C}$, the area of failure with no impact is only dictated by pattern $3 b$ (or $3 a$ ). As pattern $1 b$ is a pattern with similar dynamic properties to $3 b$, it is no surprise that the upper lobe of the area of failure with no impact for case A is very close to the boundary of the failure area with no impact for case C. It can then be considered that qualitatively the boundary of the no impact area for case $\mathrm{C}$ is translated for case $\mathrm{A}$ to the boundary of the upper lobe as indicated in Figure 13, which will be referred to as $L_{1}$.

Similarly, it can be considered that the boundary of the area of failure without impact for case B is translated for case A to the line that separates the red and yellow regions of Figure 11(b) from the green and blue, which will be referred to as $L_{2}$. Within the region defined by the translated lines, $L_{1}$ and $L_{2}$ the single rocking body cases indicate that the upper body is expected to topple to the right while the system of bodies rocking as one is expected to survive or topple to the left. Amongst the two types of failure it is substantially easier for the top body to topple to the right, as both bodies initially rotate towards the right. It is hence reasonable that within that region it is the top body that fails first, without an impact occurring in the bottom interface. This region is further separated 
depending on whether there is an impact on the top interface. The bottom part corresponds to the lower lobe of the area of the failure with no impact that was observed in Figure 12(a).

While there are other regions in the diagram that inherit properties from the simplified single rocking cases B and C, the case of stacked rocking bodies exhibits richer dynamics and it would both erroneous and non conservative to try to approximate the behaviour of the stacked system by either of the two simplified single rocking cases.

As can be seen in 11(a) for the same frequency of the input pulse, different types of failure are encountered as the amplitude changes Examples of those are shown in Figure 14 for $\omega_{u} / p=2$. When $u_{0} /(\alpha g)=1.81$ the system fails with impact in both interfaces (Figure 11(a)) and block 2 topples (Figure 11(b)). The time histories for this case are illustrated in Figure 14(a) where the rotations $\theta_{1}$ and $\theta_{2}-\theta_{1}$ indicate that the bottom interface is activated during four impacts before block 2 topples after a single impact occurring at the top interface. For $u_{0} /(\alpha g)=2.4$, the system only experiences impacts with the active interface 1 and block 2 topples without an impact activating interface 2. The time history for this case is illustrated in Figure 14(b). As the amplitude is increased to $u_{0} /(\alpha g)=4.01$, the pattern of failure with no impact is achieved and block 1 fails thus the whole system fails. The time history for this case is shown in Figure 14(c). Finally, the last type of failure appears for $u_{0} /(\alpha g)=6.21$ where only the top interface is activated with two impacts before overturning, Figure $14(d)$.

[FIG. 14 about here.]

Finally, while in the case of a single rocking body there are only two patterns of failure: the body toppling to the right or left, in this stacked body case there are several different patterns that may be active at the instance of failure. Those are presented in Figure 15, together with the the condition of failure that was activated.

[FIG. 15 about here.]

\section{Effect of $\lambda_{i}$}

The previous section made the assumption that $\lambda_{i}=0$. However, as discussed earlier, there is an uncertainty over the locations of the vertical impulses and the values of $\lambda_{i}$. This section examines how different values of $\lambda_{i}$ would affect the stability of the system of stacked rocking bodies when subjected to sinusoidal pulse type ground accelerations.

The values of $\lambda_{i}$ can take values between 0 and 1 . Figure 16 represents a sequence of stability diagrams in which the values of $\lambda_{2}$ and $\lambda_{1}$ have been increased. All cases will be compared to the case of conserving angular momentum with respect to the future rocking corners, i.e., $\lambda_{1}=\lambda_{2}=0$ (Figure 11). 
[FIG. 16 about here.]

Similarly to a single rocking body (Chatzis et al., 2016), a significant increase of the area of failure occurs as $\lambda_{1}$ is increased while retaining $\lambda_{2}=0$, as in the case of Figures 16(a) and 16(b). Similarly the stability diagram is affected when only $\lambda_{2}$ is increased as in Figures 16(c) and 16(d). When either $\lambda_{1}$ or $\lambda_{2}$, or both are substantially increased to values near unity as in Figures $16(\mathrm{~b})$ and $16(\mathrm{~d})$, not only are the failure areas increased but there appear several areas in the diagram with multiple transitions between survival and failure. In these areas the outcome of the body is highly sensitive to the amplitude and frequency of the input, similar to the observations made in (Plaut et al., 1996; Chatzis and Smyth, 2013) for rocking systems with increased number of impacts. Time histories for $\theta_{1}$ and $\theta_{2}-\theta_{1}$ are shown in Figures 17(a) and Figures $17(\mathrm{~b})$ when $\omega_{u} / p=12$ and $u_{0} /(\alpha g)=5.25$. It is clear in Figure 17 that the assumed values of both $\lambda_{i}$ have a significant effect in the time history responses of the system. An additional effect in comparison to the single rocking body case studied in Chatzis et al. (2016) is that the $\lambda_{i}$ also affect the contact interfaces that become active during the excitation.

\section{[FIG. 17 about here.]}

In this problem the increased number of impacts is due to the change of the energy exchanged between the two bodies and the energy lost by the system to the ground, which are affected by $\lambda_{2}$ and $\lambda_{1}$ respectively. While these energies also depend on the pre-impact velocities, in general higher values of $\lambda_{1}$ and $\lambda_{2}$ lead to less loss of energy per impact. This can be illustrated by defining an average total reduction of energy factor for the system, $r_{t}$, as:

$$
r_{t}=\sum_{i=1}^{N} \frac{\frac{T_{1_{i}}^{+}+T_{2_{i}}^{+}}{T_{1_{i}}^{-}+T_{2_{i}}^{-}}}{N}
$$

where $N$ is the number of impacts during the response of the system and $T_{j_{i}}^{ \pm}$is the kinetic energy of body $j$ for impact $i$ before ${ }^{-}$and after ${ }^{+}$the impact.

The values of $r_{t}$ for a range of values of $u_{0} /(\alpha g)$ and $\omega_{u} / p$ and for $\lambda_{1}=\lambda_{2}=0$ and $\lambda_{1}=0.6, \lambda_{2}=0.3$ are shown in Figures 18(a) and 18(b) respectively. Both cases are compared with their corresponding boundaries between survival and failure. Note that the white color in Figure 18 corresponds to cases where the system fails without impact. Therefore, $r_{t}$ cannot be defined.

[FIG. 18 about here.]

A comparison of Figure 18 with the corresponding stability diagrams in Figures 11 and 16(b) shows a good correspondence between the areas of high values of $r_{t}$, indicating small loss of energy, and failure. 
Stability diagrams for subjecting the system to double pulses are shown in Figure 19(a) for $\lambda_{1}=\lambda_{2}=0$, and Figure 19(b) for $\lambda_{1}=0.6$ and $\lambda_{2}=0.3$. As expected double pulses result in increasing the areas of failure in comparison to the single pulse case, even when both $\lambda_{i}=0$. Figure 19(b) suggests that the effect of the assumed values of $\lambda_{i}$ becomes of increasing importance as the number of pulse is increased and results to even further increasing the area of failure. A comparison between Figures 19(a) and 19(b), shows that the assumption of retaining angular momentum with respect to the future rocking corners can severely underestimate the tendency of the system of stacked bodies to fail for a double pulse excitation. Similar results can be obtained for further increasing the number of pulses.

[FIG. 19 about here.]

\section{Effect of body dimensions.}

The model of stacked rocking bodies often has applications to museums where the bottom body corresponds to a pedestal with the upper body being a museum artifact. A design decision that often has to be taken in a museum has to do with the dimensions of the pedestal for a given artifact. This section will investigate the effect of the combined stability of the system for artifacts and pedestals of different size. Three different combinations of bottom and top bodies are examined, as summarised in Table 4. For the first two cases, the bottom body is kept the same with top body changing, corresponding to a situation where different artifacts are placed on the same pedestal. For the last two cases the dimensions of the bottom body are varied with the top body being the same; this would correspond to an investigation of using different pedestals for the same artifact. It will be assumed that both bodies have the same density and are not hollow. (The latter assumption may not necessarily be true for museum pedestals, some of which have hollow compartments.)

\section{[TABLE 4 about here.]}

The three cases are subjected to a single cycle sinusoidal pulse ground acceleration as defined in equation (19) varying the frequency $\omega_{u}$ and the amplitude $u_{0}$. Different values of $\lambda_{i}$ are considered.

The stability diagrams for the three cases are presented in Figure 20, where the horizontal and vertical axes are normalised with $p=6.8215$ and $\alpha=0.3218$ corresponding to Block 2 for cases 2 and 3. In each figure the lines presented correspond to the boundaries between survival and failure.

\section{[FIG. 20 about here.]}

Figure 20(a) presents the boundaries of survival and failure for the three cases, when $\lambda_{1}=\lambda_{2}=0$. Comparing cases 1 and 2, where block 1 has the same aspect ratio, case 2 appears overall as a safer solution. There are, however, 
combinations of $\omega_{u} / p$ and $u_{0} /(\alpha g)$ in which case 2 results in failure while case 1 results in survival e.g, for $\omega_{u} / p=7.45$ and $u_{0} /(\alpha g)=6.55$. Figure 20(b) shows the boundaries between failure and survival when $\lambda_{1}=0.6$ and $\lambda_{2}=0$. Contrasting with the case of $\lambda_{i}=0$, case 1 now results in fewer pairs of $u_{0}, \omega_{u}$ that result in failure than case 2. However, there are still combinations of $\omega_{u} / p$ and $u_{0} /(\alpha g)$ where this situation reverses. Figure 20(c) illustrates the boundaries of failure and survival when $\lambda_{1}=0$ and $\lambda_{2}=0.6$. Similar to the case of $\lambda_{i}=0$, case 2 appears the safer solution as $\lambda_{2}$ is increased. When $\lambda_{1}=0.3$ and $\lambda_{2}=0.6$, Figure 20(d), there is no clear trend of one of the two solutions resulting in less failure. Hence, for a specific set of $u_{0}$ and $\omega_{u}$ the safest artifact, amongst the two studied here, depends on the values of $\lambda_{i}$. Equally, the optimal body in terms of decreasing the failures observed for the majority of $\omega_{u}$ and $u_{0}$ changes depending on the values of $\lambda_{i}$. However, as in the previous section, for a given case it appears that an optimal situation occurs if $\lambda_{1}=\lambda_{2}=0$. This would favour the use of absorbing materials in both interfaces, or the placement of feet on the pedestal.

Comparing now the cases where the dimensions of the pedestal are varied (case 2 and 3) it can be seen from Figure 20(a) that similar trends can be observed. While for $\lambda_{1}=\lambda_{2}=0$ case 3 appears to be safer for the majority of the pairs of $\omega_{u}, u_{0}$, there are combinations for which case 3 fails and case 2 survives, e.g., $\omega_{u} / p=11.89$ and $u_{0} /(\alpha g)=10.01$. Increasing the values of $\lambda_{i}$, Figures 20(b), 20 (c) and 20(d), results in case 3 becoming safer than case 2 for most combinations of $\omega_{u}$ and $u_{0}$. Note that in these three Figures there are also combinations of $\omega_{u} / p$ and $u_{0} /(\alpha g)$ where case 3 fails and case 2 survives. Hence, again the optimal pedestal for a given artifact is a function of the properties of the excitation $\left(u_{0}\right.$, $\omega_{u}$ ) and the interface properties $\lambda_{1}$ and $\lambda_{2}$. Again however a decrease of the values of $\lambda_{i}$ appears to improve each solution, i.e., either placing feet on the bottom of the pedestal or absorbing materials at both interfaces improves the overall stability of the system regardless of the pedestal chosen.

An alternative approach is to fix the pedestal to the ground, in which case the artifact behaves as a single rocking body. For this aim, the stability diagram for a single rocking body with the same dimensions as block 2 for cases 2 and 3 is compared with the stability diagrams of cases 2 and 3 in Figure 21.

[FIG. 21 about here.]

Although there are some pairs of $\omega_{u}, u_{0}$ where case 3 appears to be safer than fixing the pedestal to the ground, the latter case appears to be safer for a wide range of values of $\omega_{u}, u_{0}$. This is true for both the case of $\lambda_{1}=\lambda_{2}=0$, Figure 21(a), and for higher values of $\lambda_{2}$, e.g. $\lambda_{2}=0.6$ as in Figure 21(b). While, for the specific artifact it appears that fixing the pedestal to the ground is a favourable solution, in practice this has some limitations, such as the loss of mobility which is often a desirable feature in museums. Additionally, the pedestal was considered in the analysis as rigid, when in practice its flexibility may cause the acceleration at its top to be amplified in comparison to the input at the ground. Furthermore, 
in a museum there are several excitations that deviate substantially from the form of a single cycle sinusoidal base acceleration investigated here (Smyth et al., 2016). For example, accidental impact of visitors with the pedestal is a type of excitation that should be investigated where a non-fixed pedestal may potentially dissipate part of that energy by sliding.

\section{STABILITY OF STACKED BODIES SUBJECTED TO SIMULATED EARTHQUAKES}

Generally, ground motions cannot be approximated with sinusoidal pulses. There is a need to study the effect of the propagation of the uncertainty of $\lambda_{1}$ and $\lambda_{2}$ on the stability of rocking bodies subjected to earthquakes. In this section, artificially generated earthquakes are used. To this end the Spectral Representation method defined in (Shinozuka and Deodatis, 1991) is used together with the Kanai-Tajimi, Clough-Penzien spectrum (Clough and Penzien, 1975):

$$
G(\omega)=S_{0} \frac{\left(1+4 \xi_{g}^{2}\left(\frac{\omega}{\omega_{g}}\right)^{2}\right)}{\left(1-\left(\frac{\omega}{\omega_{g}}\right)^{2}\right)^{2}+4 \xi_{g}^{2}\left(\frac{\omega}{\omega_{g}}\right)^{2}} \frac{\left(\frac{\omega}{\omega_{f}}\right)^{4}}{\left(1-\left(\frac{\omega}{\omega_{f}}\right)^{2}\right)^{2}+4 \xi_{f}^{2}\left(\frac{\omega}{\omega_{f}}\right)^{2}}
$$

where $G \omega$ is the spectral density of the acceleration for a frequency $\omega, S_{0}$ denotes the ground motion intensity, $\omega_{g}$ and $\xi_{g}$ are the natural frequency and damping ratio of the ground, and $\omega_{f}$ and $\xi_{f}$ are positive parameters related to a high-pass filter that ensures finite velocities when $\omega=0$. To introduce the non-stationarity of the process, a time modulation function $\varphi(t)$ developed by (Jennings et al., 1968) is applied.

$$
\varphi(t)=\left\{\begin{array}{ll}
t / t_{1} & t<t_{1} \\
1 & t_{1} \leq t \leq t_{2} \\
e^{-\beta\left(t-t_{2}\right)} & t>t_{2}
\end{array}\right\}
$$

The generated time histories, where the properties of the filter and envelope are chosen as: $\omega_{g}=15 \mathrm{rad} / \mathrm{s}, \xi_{g}=0.6, \omega_{f}=1.5 \mathrm{rad} / \mathrm{s}, \xi_{f}=0.6, t_{1}=1 \mathrm{~s}$, $t_{2}=9 \mathrm{~s}$ and $\beta=0.2$, corresponding to a firm soil. The probability of failure is calculated by varying $\sqrt{S_{0}}$ as a measure of intensity and generating a suitable number of records. To this end, 5000 earthquakes are generated for $S_{0}=1$ and are later uniformly scaled by multiplying the amplitudes by $\sqrt{S_{0}}$. The probability is calculated using a Monte Carlo analysis as the sum of records for which failure occurs over the total number of records per value of $S_{0}$. Depending on the assumed value of $\lambda_{i}$, a different curve is produced as shown in Figure 22 for a system of stacked bodies with dimensions $h_{1}=0.5565 \mathrm{~m}, b_{1}=0.3970 \mathrm{~m}, h_{2}=0.3 \mathrm{~m}$ and $b_{2}=0.15 \mathrm{~m}$. The body dimensions would correspond to the bodies comprising a large monumental statue on a pedestal. They are also chosen as an example of a body where both $l a m b d a_{i}$ have an equally important effect on the stability of the system. 
[FIG. 22 about here.]

As expected,it can be seen in Figure 22, as either $\lambda_{i}$ is increased the probability of failure for a given value of $\sqrt{S_{0}}$ increases. While in this specific system $\lambda_{2}$ appears to have a greater effect than $\lambda_{1}$, there are examples where this is reversed. For this system and for an earthquake type of excitation, the trend that has been observed in the previous examples of $\lambda_{i}=0$ corresponding to a smaller probability of failure is observed again. Hence, as for a single rocking body, the choice of $\lambda_{i}=0$ is the least conservative assumption, and modifications that cause a reduction in the values of $\lambda_{i}$ in both interfaces are a desirable solution.

\section{CONCLUSIONS}

This paper extends the discussion in (Chatzis et al., 2016) for the effect of the location of the vertical impulses during impact, to the case of two stacked rocking bodies. The paper discusses the assumptions involved in modelling the loss of energy during impact in hard impact models, when sliding, uplift, ground deformability and the three dimensional nature of the response are neglected. The location of the vertical impulses during impact can be applied at any point between the post-impact rocking corner of a block and the midpoint of its base, without violating any of the assumptions of the model. This paper considers the effects of this vertical impulse acting on interface $i$ at a distance $\lambda_{i} b_{i}, \lambda_{i} \in[0,1]$, from the future rocking corner.

To that end the momentum equations and transitions between patterns during impacts that were first derived in the work of (Spanos et al., 2001) assuming $\lambda_{1}=\lambda_{2}=0$ were re-derived. It was highlighted that the same non-tensile stresses assumption for the interface that defines the transitions without impact, implies that the vertical impulses have to be applied between the corner points of the rocking body above the interface. Additionally, it was shown that even in the original work of (Spanos et al., 2001) there are situations where specific non-zero values of $\lambda_{i}$ must be adopted so that the post-impact pattern is permissible. This paper argues that such cases would instead result in loss of contact at one of the two interfaces, and hence free flight of part of the system. When such cases occur, a model that does not take into account such patterns should not be trusted.

The stability of the system subjected to simple single and double sinusoidal acceleration pulses was studied. As for the case of a single rocking body, it has shown that the common assumption in the literature, that $\lambda_{i}=0$, is the least conservative scenario, as increasing the values of $\lambda_{i}$ expands the area of failure. Higher values of $\lambda_{i}$ result in multiple impacts prior to failure and, similar to the case of multiple pulses, introduce areas of $\omega_{u}$ and $u_{0}$ for which the stability of the body becomes sensitive on the exact values.

A practical study of the failure of different stacked bodies was performed. This study is applicable to, for example, museums where artifacts are placed on pedestals. For the systems studied, there is not a single solution which is the safest for all the combinations of $\omega_{u} / p$ and $u_{0} /(\alpha g)$. The optimal solution 
appears to be a function of the values of $\lambda_{i}$ for the interfaces, which should be taken into account for design purposes. However, when $\lambda_{i}=0$, the areas of failure tend to decrease. Hence, it appears that the use of energy absorbing materials in the contact interfaces, or of feet at the bottom of the bodies, whenever possible, would result in usually safer solutions in terms of failure of the system through toppling.

Finally, the influence of $\lambda_{1}$ and $\lambda_{2}$ on the probability of failure of a system of stacked rocking bodies subjected to artificially generated earthquakes was examined. Again, it was shown that the assumption of $\lambda_{1}=0$ and $\lambda_{2}=0$ may result in underestimation of the probability of failure.

This paper shows the need for taking into account the uncertainty related to the assumption of how energy is lost during impacts, as expressed through the range of allowable values of $\lambda_{i}$. However, even with suitable values of $\lambda_{i}$ for the interfaces, this model of stacked rocking bodies may not approximate the physical response of a real system. This is because sliding in such systems is almost inevitable for large rotations. Equally, as most of these objects have an almost square base, three dimensional rocking is likely to occur.

Even if these assumptions had a minimal effect, there would still be an uncertainty over the values of $\lambda_{i}$ that would influence the stability of the system. The sources of this uncertainty increase with the number of interfaces that may become active in a rocking system. This is a modelling uncertainty due to not modelling the contact interface property in the IPM. Hence, when expanding the IPM into multiple bodies, or dimensions, the researchers should consider this effect in their conclusions, together with the likelihood that the usual assumption of conserving momentum with respect to the future rocking corners may not activated all the possible modes of response. If it is desired to retain this assumption then an effort should be made to mechanically modify the bodies, so that the impulses are forced to pass through the corners, for example by placing feet on the components of the rocking system as suggested in (Chatzis and Smyth, 2012c; Chatzis et al., 2016). Alternatively the authors suggest the use of models that take into account the deformability of the contact interface, sliding, uplift and the three-dimensional nature of the response.

\section{ACKNOWLEDGEMENTS}

The first two authors would like to acknowledge the financial support of the EC, FP7-PEOPLE-2013, Marie Curie, Career Integration Grant, RERCSGM, project number 618359 .

\section{REFERENCES}

Allen, R. H., Oppenheim, I. J., Parker, A. R., and Bielak, J. (1986). "On the dynamic response of rigid body assemblies." Earthquake Engineering \&f Structural Dynamics, 14(6), 861-876. 
Chatzis, M. and Smyth, A. (2013). "Preliminary investigation of the random in the outcome of a die throw." Proceedings ICOSSAR 2013 11th International Conference on Structural Safety and Reliability.

Chatzis, M. N., Garcia-Espinosa, M., and Smyth, A. W. (2016). "Examining the energy loss in the inverted pendulum model for rocking bodies." Journal of Engineering Mechanics.

Chatzis, M. N. and Smyth, A. W. (2012a). "The 3d dynamics of a rigid body with wheels on a moving base." Journal of Engineering Mechanics, doi: 10.1061/(ASCE)EM.1943-7889.0000456.

Chatzis, M. N. and Smyth, A. W. (2012b). "Modeling of the 3d rocking problem." International Journal of Non-Linear Mechanics, 47, 85-98.

Chatzis, M. N. and Smyth, A. W. (2012c). "Robust modeling of the rocking problem." Journal of Engineering Mechanics, 3, 247-262.

Clough, R. W. and Penzien, J. (1975). Dynamics of Structures. McGraw-Hill.

Dimitrakopoulos, E. G. and Giouvanidis, A. I. (2015). "Seismic response analysis of the planar rocking frame." Journal of Engineering Mechanics, 141(7), 04015003.

Dormand, J. and Prince, P. (1980). "A family of embedded runge-kutta formula." Journal of Computational and Applied Mathematics, 6(1), 19 - 26.

ElGawady, M. A., Ma, Q., Butterworth, J. W., and Ingham, J. (2011). "Effects of interface material on the performance of free rocking blocks." Earthquake Engineering \&5 Structural Dynamics, 40(4), 375-392.

FEMA (2006). Document Control Procedures Manual (September).

Greenwood, D. T. (2003). Advanced dynamics. Cambridge University Press.

Harvey Jr, P. (2017). "Behavior of a rocking block resting on a rolling isolation system." Journal of Engineering Mechanics, 143(8), 04017045.

Housner, G. W. (1963). "The behavior of inverted pendulum structures during earthquakes." Bulletin of the Seismological Society of America, 53, 403-417.

Jennings, P. C., Housner, G. W., and Tsai, N. C. (1968). "Simulated earthquake motions.

Kounadis, A. N., Papadopoulos, G. J., and Cotsovos, D. M. (2012). "Overturning instability of a two-rigid block system under ground excitation." ZAMMJournal of Applied Mathematics and Mechanics/Zeitschrift für Angewandte Mathematik und Mechanik, 92(7), 536-557. 
Lipscombe, P. R. and Pellegrino, S. (1991). "Free rocking of prismatic bloocks." Engineering Mechanics, 119, 1387-1410.

Makris, N. and Vassiliou, M. F. (2014). "Are some top-heavy structures more stable?." Journal of Structural Engineering, 140(5), 06014001.

Nikfar, F. and Konstantinidis, D. (2017). "Shake table investigation on the seismic performance of hospital equipment supported on wheels/casters." Earthquake Engineering \& Structural Dynamics, 46(2), 243-266.

Palmeri, A. and Makris, N. (2008). "Response analysis of rigid structures rocking on viscoelastic foundation." Earthquake Engineering and Structural Dynamics, 37, 1039-1063.

Plaut, R., Fielder, W., and Virgin, L. (1996). "Fractal behavior of an asymmetric rigid block overturning due to harmonic motion of a tilted foundation." Chaos, Solitons \& Fractals, 7(2), 177-196.

Psycharis, I. N. (1990). "Dynamic behaviour of rocking two-block assemblies." Earthquake Engineering 83 Structural Dynamics, 19(4), 555-575.

Psycharis, I. N. and Jennings, P. C. (1983). "Rocking of slender rigid bodies allowed to uplift.." Earthquake Eng Struct Dyn, 11(11), 57-76.

Shampine, L. F. and Gordon, M. K. (1975). Computer solution of ordinary differential equations: the initial value problem. WH Freeman San Francisco.

Shenton, H. and Jones, N. (1991). "Base excitation of rigid bodies. 1: Formulation." Journal of Engineering Mechanics, 117, 2286-2306.

Shinozuka, M. and Deodatis, G. (1991). "Simulation of stochastic processes by spectral representation." American Society of Mechanical Engineering, 44(4), 191-204.

Smyth, A. W., Brewick, P., Greenbaum, R., Chatzis, M., Serotta, A., and Stünkel, I. (2016). "Vibration mitigation and monitoring: a case study of construction in a museum." Journal of the American Institute for Conservation, 55(1), 32-55.

Spanos, P. D., Roussis, P. C., and Politis, N. P. (2001). "Dynamic analysis of stacked rigid blocks." Soil Dynamics and Earthquake Engineering, 21(7), 559 -578 .

Vassiliou, M. F. and Makris, N. (2012). "Analysis of the rocking response of rigid blocks standing free on a seismically isolated base." Earthquake Engineering $\mathcal{E}$ Structural Dynamics, 41(2), 177-196.

Voyagaki, E., Psycharis, I., and Mylonakis, G. (2014). "Complex response of a rocking block to a full-cycle pulse." Journal of Engineering Mechanics, 140(6), 04014024 . 
Wittich, C. E. and Hutchinson, T. C. (2016). "Experimental modal analysis and seismic mitigation of statue-pedestal systems." Journal of Cultural Heritage, 20, 641-648.

Yim, C.-S., Chopra, A. K., and Penzien, J. (1980). "Rocking response of rigid blocks to earthquakes." Earthquake Engineering 85 Structural Dynamics, 8(6), $565-587$.

Zhang, H., Brogliato, B., and Liu, C. (2012). "Study of the planar rocking-block dynamics with coulomb friction: Critical kinetic angles." Journal of Computational and Nonlinear Dynamics, 8`(2).

Zhang, J. and Makris, N. (2001). "Rocking response of free-standing blocks under cycloidal pulses.." Journal of Engineering Mechanics, 127(5), 473-483.

Zulli, D., Contento, A., and Egidio, A. D. (2012). "3d model of rigid block with a rectangular base subject to pulse-type excitation." International Journal of Non-Linear Mechanics, 47(6), 679 - 687.

\section{Appendices}

\section{MOMENTUM EQUATIONS}

\section{Pattern 1}

From pattern $1 \mathrm{a}$ to $2 \mathrm{~b}$,

$$
\begin{aligned}
A_{1} & =I_{O_{1}}-\left(2-\lambda_{1}\right) m_{1} b_{1}^{2}+m_{2} l R_{2} \cos \left(\theta_{2}+\beta-\alpha_{2}\right) & & A_{2}=m_{2} l R_{2} \cos \left(\theta_{2}+\beta-\alpha_{2}\right) \\
& +2 m_{2} l h_{1} \cos \beta-\left(\left(1-\lambda_{1}\right) b_{1}+b_{2}\right) m_{2} l \sin \beta & & \\
B_{1} & =I_{O_{2}}+2 m_{2} R_{2} h_{1} \cos \left(\alpha_{2}-\theta_{2}\right) & & B_{2}=I_{O_{2}} \\
& -\left(\left(1-\lambda_{1}\right) b_{1}+b_{2}\right) m_{2} R_{2} \sin \left(\alpha_{2}-\theta_{2}\right) & & \\
C_{1} & =I_{O_{1}}-\lambda_{1} m_{1} b_{1}^{2}+m_{2} l^{\prime} R_{2} \cos \left(\theta_{2}-\beta^{\prime}-\alpha_{2}\right) & & C_{2}=m_{2} l^{\prime} R_{2} \cos \left(\theta_{2}-\beta^{\prime}-\alpha_{2}\right) \\
& +2 m_{2} l^{\prime} h_{1} \cos \beta^{\prime}+\left(\left(1-\lambda_{1}\right) b_{1}+b_{2}\right) m_{2} l^{\prime} \sin \beta^{\prime} & & \\
D_{1} & =I_{O_{2}}+2 m_{2} R_{2} h_{1} \cos \left(\alpha_{2}-\theta_{2}\right) & & D_{2}=I_{O_{2}} \\
& -\left(\left(1-\lambda_{1}\right) b_{1}+b_{2}\right) m_{2} R_{2} \sin \left(\alpha_{2}-\theta_{2}\right) & &
\end{aligned}
$$

From 1a to $4 \mathrm{a}: \dot{\theta}_{1}^{+}=0$, hence,

$$
\begin{aligned}
\dot{\theta}_{2}^{+} & =\frac{A_{2}}{D_{2}} \dot{\theta}_{1}^{-}+\dot{\theta}_{2}^{-} \\
\lambda_{1} & =\frac{A_{3} \dot{\theta}_{1}^{-}+B_{3} \dot{\theta}_{2}^{-}+C_{3} \dot{\theta}_{2}^{+}}{D_{3} \dot{\theta}_{1}^{-}+E_{3} \dot{\theta}_{2}^{-}+F_{3} \dot{\theta}_{2}^{+}}
\end{aligned}
$$


where,

$$
\begin{aligned}
& A_{3}=-\left(I_{O 1}-2 m_{1} b_{1}^{2}+m_{2} l R_{2} \cos \left(\theta_{2}+\beta-\alpha_{2}\right)+2 m_{2} l h_{1} \cos \beta-\left(b_{1}+b_{2}\right) m_{2} l \sin \beta\right) \\
& B_{3}=-\left(I_{O 2}+2 m_{2} R_{2} H_{1} \cos \left(\alpha_{2}-\theta_{2}\right)-\left(b_{1}+b_{2}\right) m_{2} R_{2} \sin \left(\alpha_{2}-\theta_{2}\right)\right) \\
& C_{3}=I_{O 2}+2 m_{2} R_{2} h_{1} \cos \left(\alpha_{2}-\theta_{2}\right)-\left(b_{1}+b_{2}\right) m_{2} R_{2} \sin \left(\alpha_{2}-\theta_{2}\right) \\
& D_{3}=m_{1} b_{1}^{2}+b_{1} m_{2} l \sin \beta \\
& E_{3}=b_{1} m_{2} R_{2} \sin \left(\alpha_{2}-\theta_{2}\right) \\
& F_{3}=-\left(b_{1} m_{2} R_{2} \sin \left(\alpha_{2}-\theta_{2}\right)\right.
\end{aligned}
$$

By symmetry this analysis holds for the transition from pattern $1 \mathrm{~b}$ to $2 \mathrm{a}$ and $4 \mathrm{~b}$, though it should be noted that the sign of $\theta_{2}$ must change.

From pattern 1a to $2 \mathrm{a}$,

$$
\begin{array}{ll}
A_{1}=I_{O_{1}}+m_{2} l^{2}+m_{2} l R_{2} \cos \left(\alpha_{2}-\beta\right) & A_{2}=m_{2} l R_{2} \cos \left(\alpha_{2}+\beta\right)+\lambda_{2} m_{2} l b_{2} \sin \beta \\
B_{1}=I_{O_{2}}+m_{2} l R_{2} \cos \left(\alpha_{2}-\beta\right) & B_{2}=I_{G_{2}}+m_{2} R_{2}{ }^{2} \cos \left(2 \alpha_{2}\right)+\lambda_{2} m_{2} R_{2} b_{2} \sin \alpha_{2} \\
C_{1}=I_{O_{1}}+m_{2} l^{\prime 2}+m_{2} l^{\prime} R_{2} \cos \left(\alpha_{2}+\beta^{\prime}\right) & C_{2}=m_{2} l^{\prime} R_{2} \cos \left(\alpha_{2}+\beta^{\prime}\right)+\lambda_{2} m_{2} l^{\prime} b_{2} \sin \beta^{\prime} \\
D_{1}=I_{O_{2}}+m_{2} l^{\prime} R_{2} \cos \left(\alpha_{2}+\beta^{\prime}\right) & D_{2}=I_{O_{2}}-\lambda_{2} m_{2} R_{2} b_{2} \sin \alpha_{2}
\end{array}
$$

From 1a to $3 \mathrm{a}: \dot{\theta}_{2}^{+}=\dot{\theta}_{1}^{+}$

$$
\begin{aligned}
\dot{\theta}_{1}^{+} & =\frac{A_{1}}{C_{1}+D_{1}} \dot{\theta}_{1}^{-}+\frac{B_{1}}{C_{1}+D_{1}} \dot{\theta}_{2}^{-} \\
\lambda_{2} & =\frac{A_{3} \dot{\theta}_{1}^{-}+B_{3} \dot{\theta}_{2}^{-}+C_{3} \dot{\theta}_{1}^{+}}{D_{3} \dot{\theta}_{1}^{-}+E_{3} \dot{\theta}_{2}^{-}+F_{3} \dot{\theta}_{1}^{+}}
\end{aligned}
$$

where,

$$
\begin{array}{ll}
A_{3}=m_{2} l R_{2} \cos \left(\alpha_{2}+\beta\right) & B_{3}=I_{G 2}+m_{2} R_{2}^{2} \cos \left(2 \alpha_{2}\right) \\
C_{3}=-\left(m_{2} l^{\prime} R_{2} \cos \left(\alpha_{2}+\beta^{\prime}\right)+I_{O 2}\right) & D_{3}=-\left(m_{2} l b_{2} \sin \beta\right) \\
E_{3}=-\left(m_{2} R_{2} b_{2} \sin \alpha_{2}\right) & F_{3}=m_{2} l^{\prime} b_{2} \sin \beta^{\prime}-m_{2} R_{2} b_{2} \sin \alpha_{2}
\end{array}
$$

By symmetry this analysis holds for the transition from pattern $1 \mathrm{~b}$ to $2 \mathrm{~b}$ or $3 \mathrm{~b}$.

\section{Pattern 2}

From pattern $2 \mathrm{a}$ to $1 \mathrm{~b}$, 


$$
\begin{aligned}
A_{1} & =I_{O_{1}}-\left(2-\lambda_{1}\right) m_{1} b_{1}{ }^{2}+m_{2} l^{2} & & A_{2}=m_{2} l^{\prime} R_{2} \cos \left(\theta_{2}+\alpha_{2}+\beta^{\prime}\right) \\
& +m_{2} l^{\prime} R_{2} \cos \left(\theta_{2}+\alpha_{2}+\beta^{\prime}\right)-\left(2-\lambda_{1}\right) m_{2} l^{\prime} b_{1} \sin \beta^{\prime} & & \\
B_{1} & =I_{O_{2}}+m_{2} l^{\prime} R_{2} \cos \left(\theta_{2}+\alpha_{2}+\beta^{\prime}\right) & & B_{2}=I_{O_{2}} \\
& +\left(2-\lambda_{1}\right) m_{2} R_{2} b_{1} \sin \left(\alpha_{2}+\theta_{2}\right) & & \\
C_{1} & =I_{O_{1}}-\lambda_{1} m_{1} b_{1}^{2}+m_{2} l^{2} & & C_{2}=m_{2} l R_{2} \cos \left(\theta_{2}+\alpha_{2}-\beta^{\prime}\right) \\
& +m_{2} l R_{2} \cos \left(\theta_{2}+\alpha_{2}-\beta\right)-\lambda_{1} m_{2} l b_{1} \sin \beta & & \\
D_{1} & =I_{O_{2}}+m_{2} l R_{2} \cos \left(\theta_{2}+\alpha_{2}-\beta\right) & & D_{2}=I_{O_{2}}
\end{aligned}
$$

From $2 \mathrm{a}$ to $4 \mathrm{~b}: \dot{\theta}_{1}^{+}=0$

$$
\begin{aligned}
\dot{\theta}_{2}^{+} & =\frac{A_{2}}{D_{2}} \dot{\theta}_{1}^{-}+\dot{\theta}_{2}^{-} \\
\lambda_{1} & =\frac{A_{3} \dot{\theta}_{1}^{-}+B_{3} \dot{\theta}_{2}^{-}+C_{3} \dot{\theta}_{2}^{+}}{D_{3} \dot{\theta}_{1}^{-}+E_{3} \dot{\theta}_{2}^{-}+F_{3} \dot{\theta}_{2}^{+}}
\end{aligned}
$$

where,

$$
\begin{aligned}
A_{3} & =I_{O 1}-2 m_{1} b_{1}^{2}+m_{2} l l^{2} & B_{3} & =I_{O 2}+m_{2} l^{\prime} R_{2} \cos \left(\theta_{2}+\alpha_{2}+\beta^{\prime}\right) \\
& +m_{2} l^{\prime} R_{2} \cos \left(\theta_{2}+\alpha_{2}+\beta^{\prime}\right)-2 m_{2} l \prime b_{1} \sin \beta^{\prime} & & +2 m_{2} R_{2} b_{1} \sin \left(\alpha_{2}+\theta_{2}\right) \\
C_{3} & =-\left(I_{O 2}+m_{2} l R_{2} \cos \left(\theta_{2}+\alpha_{2}-\beta\right)\right) & D_{3} & =-\left(m_{1} b_{1}^{2}+m_{2} l^{\prime} b_{1} \sin \beta^{\prime}\right) \\
E_{3} & =m_{2} R_{2} b_{1} \sin \left(\alpha_{2}+\theta_{2}\right) & F_{3} & =-m_{2} R_{2} b_{1} \sin \left(\alpha_{2}+\theta_{2}\right)
\end{aligned}
$$

By symmetry this analysis is valid for the transition from pattern $2 \mathrm{~b}$ to $1 \mathrm{a}$ or $4 \mathrm{a}$, though it should be noted that the sign of $\theta_{2}$ must change.

From pattern $2 \mathrm{a}$ to $1 \mathrm{a}$,

$$
\begin{array}{ll}
A_{1}=I_{O_{1}}+m_{2} l^{2}+m_{2} l^{\prime} R_{2} \cos \left(\alpha_{2}+\beta^{\prime}\right) & A_{2}=m_{2} l^{\prime} R_{2} \cos \left(\beta^{\prime}-\alpha_{2}\right)-\lambda_{2} m_{2} l^{\prime} b_{2} \sin \beta^{\prime} \\
B_{1}=I_{O_{2}}+m_{2} l^{\prime} R_{2} \cos \left(\alpha_{2}+\beta^{\prime}\right) & B_{2}=I_{G_{2}}+m_{2} R_{2}{ }^{2} \cos \left(2 \alpha_{2}\right)+\lambda_{2} m_{2} R_{2} b_{2} \sin \alpha_{2} \\
C_{1}=I_{O_{1}}+m_{2} l^{2}+m_{2} l R_{2} \cos \left(\alpha_{2}-\beta\right) & C_{2}=m_{2} l R_{2} \cos \left(\alpha_{2}-\beta\right)-\lambda_{2} m_{2} l b_{2} \sin \beta \\
D_{1}=I_{O_{2}}+m_{2} l R_{2} \cos \left(\alpha_{2}-\beta\right) & D_{2}=I_{O_{2}}-\lambda_{2} m_{2} R_{2} b_{2} \sin \alpha_{2}
\end{array}
$$

From $2 \mathrm{a}$ to $3 \mathrm{a}: \dot{\theta}_{2}^{+}=\dot{\theta}_{1}^{+}$

$$
\begin{aligned}
\dot{\theta}_{1}^{+} & =\frac{A_{1}}{C_{1}+D_{1}} \dot{\theta}_{1}^{-}+\frac{B_{1}}{C_{1}+D_{1}} \dot{\theta}_{2}^{-} \\
\lambda_{2} & =\frac{A_{3} \dot{\theta}_{1}^{-}+B_{3} \dot{\theta}_{2}^{-}+C_{3} \dot{\theta}_{1}^{+}}{D_{3} \dot{\theta}_{1}^{-}+E_{3} \dot{\theta}_{2}^{-}+F_{3} \dot{\theta}_{1}^{+}}
\end{aligned}
$$


where,

$$
\begin{array}{ll}
A_{3}=m_{2} l^{\prime} R_{2} \cos \left(\beta^{\prime}-\alpha_{2}\right) & B_{3}=I G 2+m_{2} R_{2}^{2} \cos \left(2 \alpha_{2}\right) \\
C_{3}=-\left(m_{2} l R_{2} \cos \left(\alpha_{2}-\beta\right)+I_{O 2}\right) & D_{3}=m_{2} l^{\prime} b_{2} \sin \beta^{\prime} \\
E_{3}=-m_{2} R_{2} b_{2} \sin \left(\alpha_{2}\right) & F_{3}=-m_{2} l b_{2} \sin \beta-m_{2} R_{2} b_{2} \sin \alpha_{2}
\end{array}
$$

0

\section{Pattern 3}

By symmetry the analysis in section Special transition cases holds for the transitions from pattern $3 \mathrm{~b}$ to $1 \mathrm{a}, 3 \mathrm{a}$ or $4 \mathrm{a}$.

\section{Pattern 4}

From pattern 4 a to $1 b$,

$$
\begin{aligned}
A_{1} & =0 & & A_{2}=0 \\
B_{1} & =I_{G_{2}}+m_{2} R_{2}\left(2 h_{1}+h_{2}\right) \cos \alpha_{2} & & B_{2}=I_{O 2}-\left(2-\lambda_{2}\right) m_{2} b_{2}{ }^{2} \\
& -\left(1-\lambda_{1}\right) m_{2} R_{2} b_{1} \sin \alpha_{2} & & \\
C_{1} & =I_{O_{1}}-\lambda_{1} m_{1} b_{1}{ }^{2}+m_{2} l\left(2 h_{1}+h_{2}\right) \cos \beta & & C_{2}=m_{2} l h_{2} \cos \beta+\left(1-\lambda_{2}\right) m_{2} l b_{2} \sin \beta \\
& +\left(1-\lambda_{1}\right) m_{2} l b_{1} \sin \beta & & \\
D_{1} & =I_{G_{2}}+m_{2} R_{2}\left(2 h_{1}+h_{2}\right) \cos \alpha_{2} & & D_{2}=I_{O_{2}}-\lambda_{2} m_{2} b_{2}{ }^{2} \\
& +\left(1-\lambda_{1}\right) m_{2} R_{2} b_{1} \sin \alpha_{2} & &
\end{aligned}
$$

From pattern $4 \mathrm{a}$ to $4 \mathrm{~b}: \dot{\theta}_{1}^{+}=0$

$$
\dot{\theta}_{2}^{+}=\frac{I_{O_{2}}-\left(2-\lambda_{2}\right) m_{2} b_{2}^{2}}{I_{O_{2}}-\lambda_{2} m_{2} b_{2}^{2}} \dot{\theta}_{2}^{-}
$$

$$
\lambda_{1}=\frac{A_{3} \dot{\theta}_{2}^{-}+B_{3} \dot{\theta}_{2}^{+}}{C_{3} \dot{\theta}_{2}^{-}+D_{3} \dot{\theta}_{2}^{+}}
$$

where,

$$
\begin{array}{lc}
A_{3}=-\left(I_{G 2}+m_{2} R_{2}\left(2 h_{1}+h_{2}\right) \cos \alpha_{2}-\right. & B_{3}=I_{G 2}+m_{2} r_{2}\left(2 h_{1}+h_{2}\right) \cos \alpha_{2}+ \\
\left.m_{2} R_{2} b_{1} \sin \alpha_{2}\right) & m_{2} R_{2} b_{1} \sin \alpha_{2} \\
C_{3}=m_{2} R_{2} b_{1} \sin \alpha_{2} & D_{3}=m_{2} R_{2} b_{1} \sin \alpha_{2}
\end{array}
$$


From pattern 4 a to $3 \mathrm{~b}, \dot{\theta}_{2}^{+}=\dot{\theta}_{1}^{+}$

971

$$
\begin{aligned}
\dot{\theta}_{1}^{+} & =\frac{B_{1}}{I_{O}-\lambda_{1} M b_{1}{ }^{2}} \dot{\theta}_{2}^{-} \\
\lambda_{2} & =\frac{A_{3} \dot{\theta}_{1}^{+}+B_{3} \dot{\theta}_{2}^{-}}{C_{3} \dot{\theta}_{2}^{-}+D_{3} \dot{\theta}_{1}^{+}}
\end{aligned}
$$

972 where,

$$
\begin{array}{ll}
A_{3}=-\left(I_{O_{2}}-2 m_{2} b_{2}^{2}\right) & B_{3}=I_{O_{2}}+m_{2} l h_{2} \cos \beta+m_{2} l b_{2} \sin \beta \\
C_{3}=m_{2} b_{2}^{2} & D_{3}=m_{2} b_{2}\left(l \sin \beta+b_{2}\right)
\end{array}
$$

973

Note that this analysis is valid for the transition of pattern $4 \mathrm{~b}$ to $1 \mathrm{a}$, $4 \mathrm{a}$ or $3 \mathrm{a}$. 

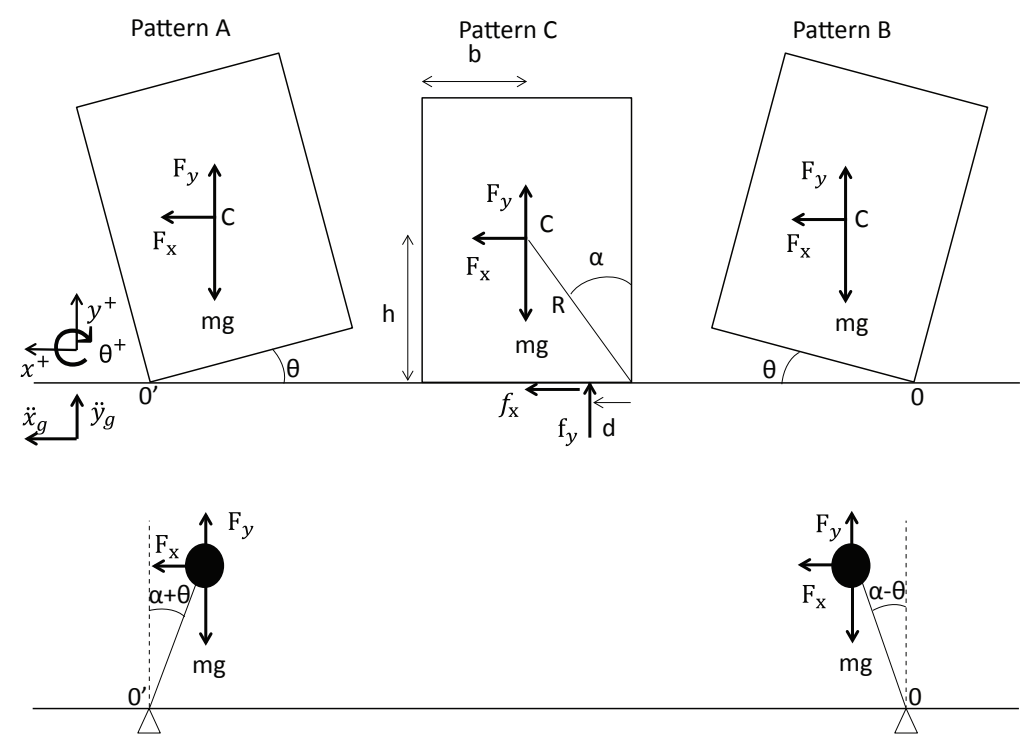

FIG. 1 - Inverted Pendulum model defined by (Housner, 1963) for a free-standing rocking block. 


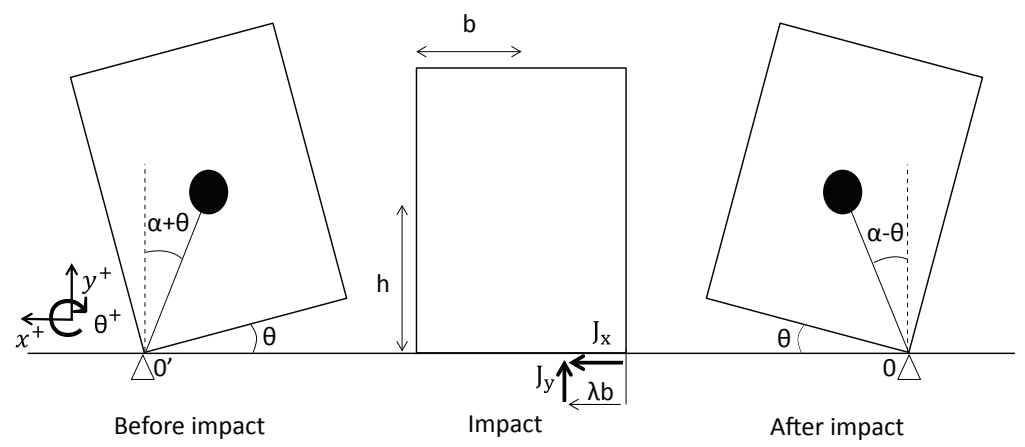

FIG. 2 - It is assumed, with any loss of generality, that the impact occurs switching from Pattern A to B. At impact the body experiences an horizontal, $J_{x}$ and vertical, $J_{y}$, impulses at $\lambda$. 


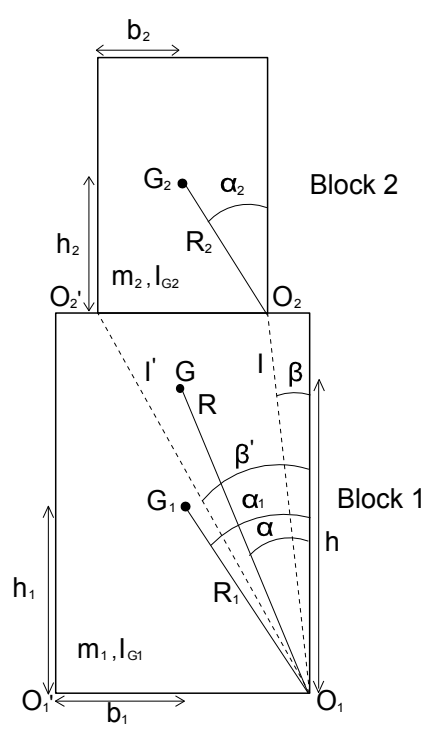

(a) Geometry

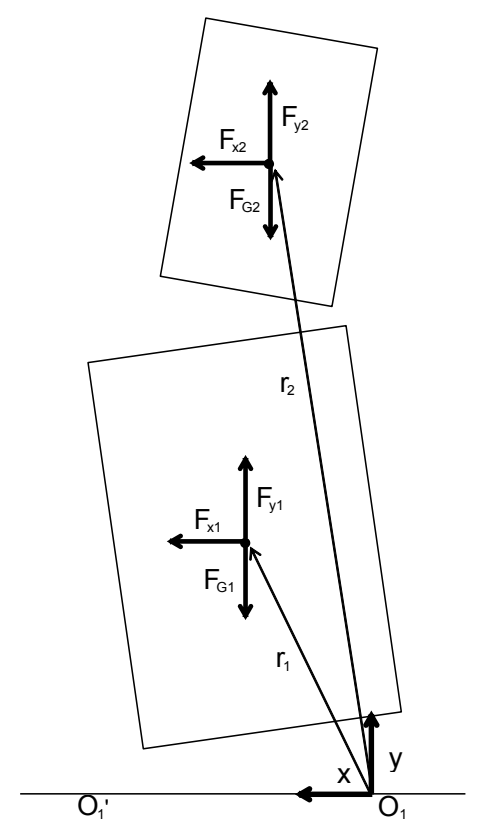

(b) Kinematics

FIG. 3 - a) Geometry of two stacked blocks. b) Forces and kinematics of the bodies. Note that this model does not allow uplifting, the separation from the ground is only for illustration reasons. 


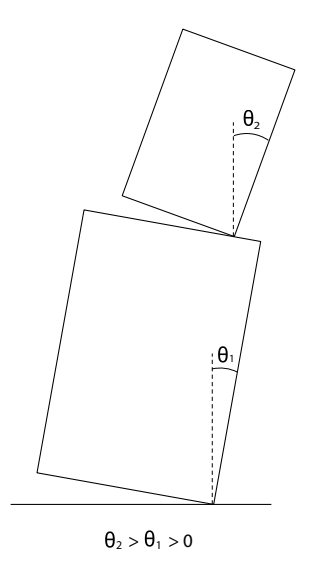

(a) Pattern 1a

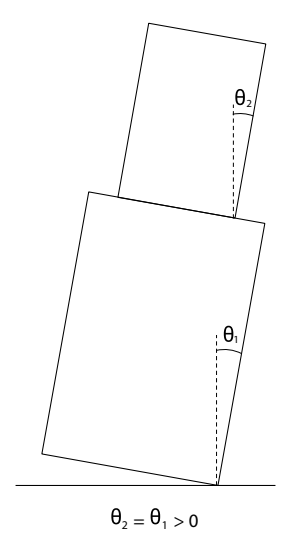

(e) Pattern 3a

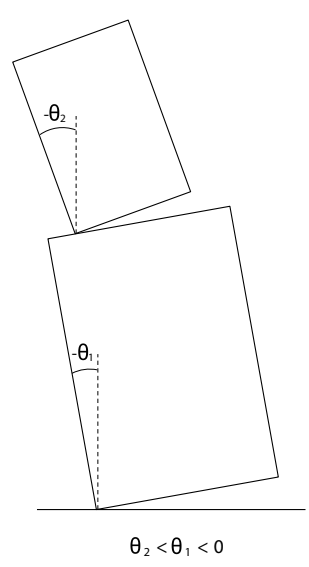

(b) Pattern 1b

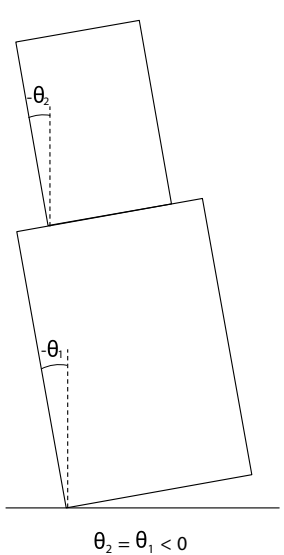

(f) Pattern 3b

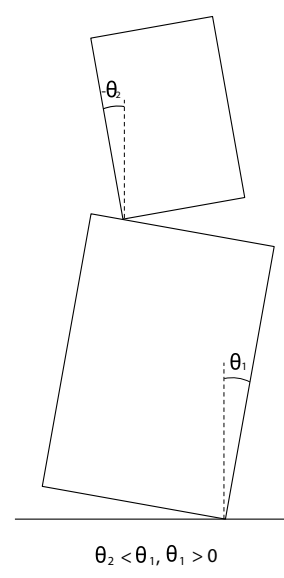

(c) Pattern 2a

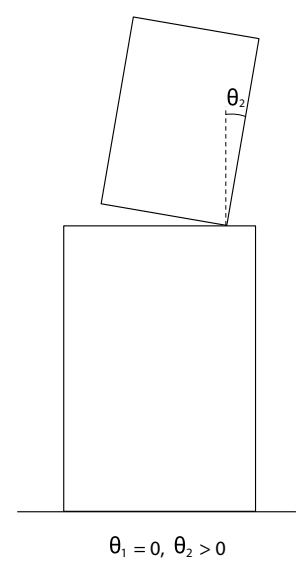

(g) Pattern 4a

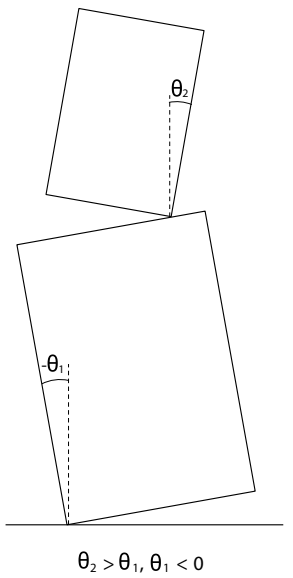

(d) Pattern 2b

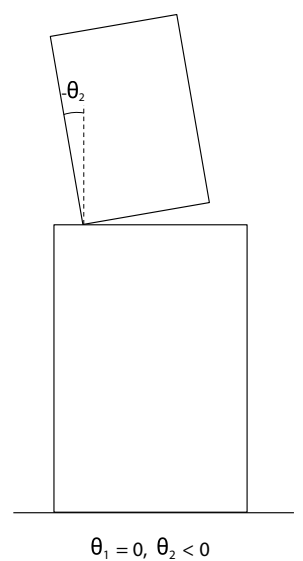

(h) Pattern 4b

FIG. 4 - All possible dynamic patterns of rocking for two stacked blocks 


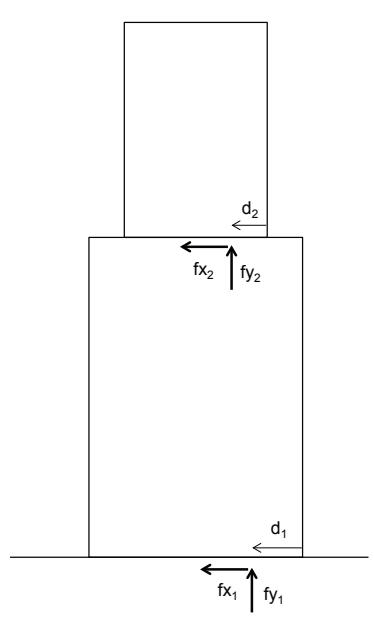

(a) Both interfaces active

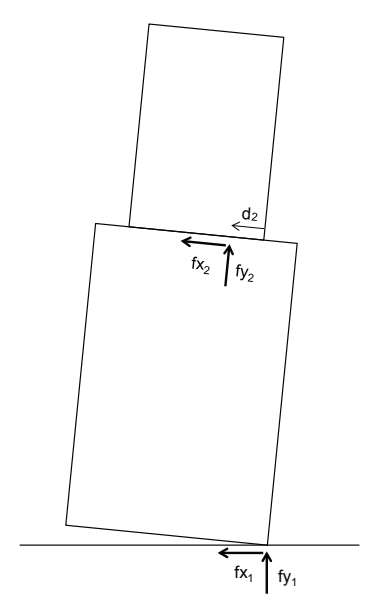

(b) Bottom interface active

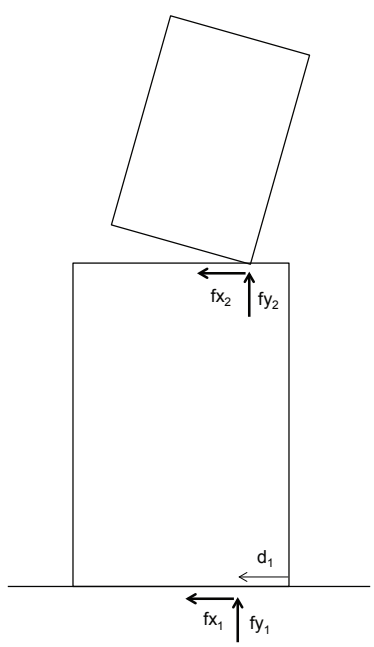

(c) Top interface active

FIG. 5 - All possible cases of the reactions forces. 


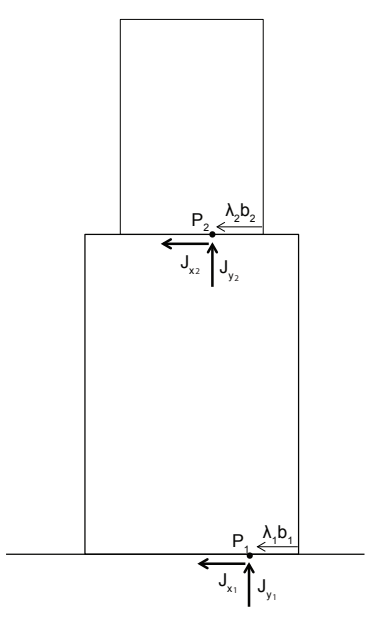

(a) Both interfaces active

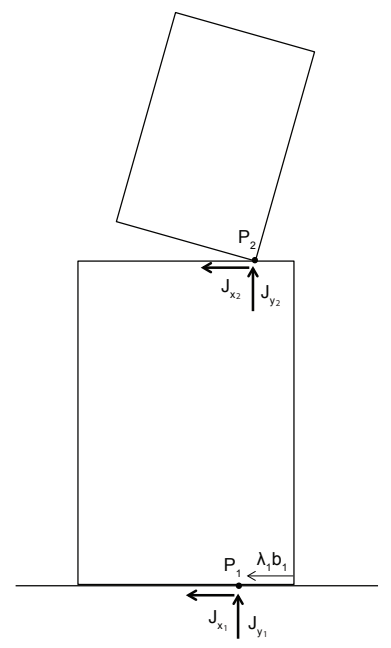

(b) Bottom interface active

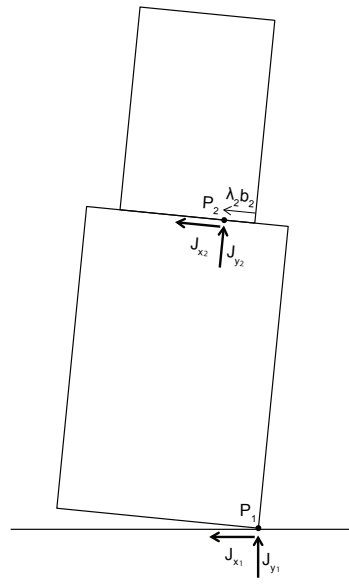

(c) Top interface active

FIG. 6 - Three characteristic cases during impact. 

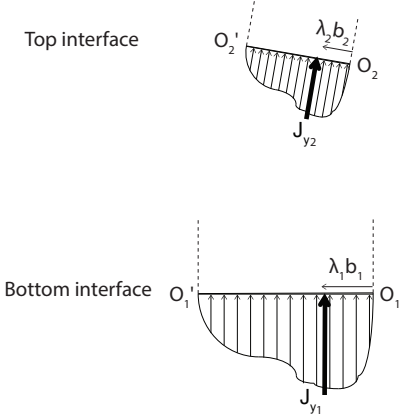

(a) Physically possible
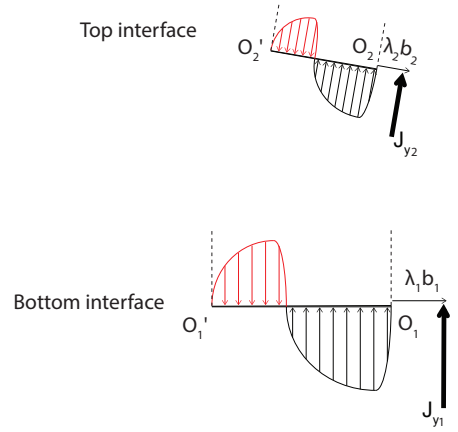

(b) Non-physically possible

FIG. 7 - a) One of the infinite admissible distributions to describe the vertical impulses during impact. b) Distribution showing the tensile stresses which are a violation of the non-tensile assumptions. 


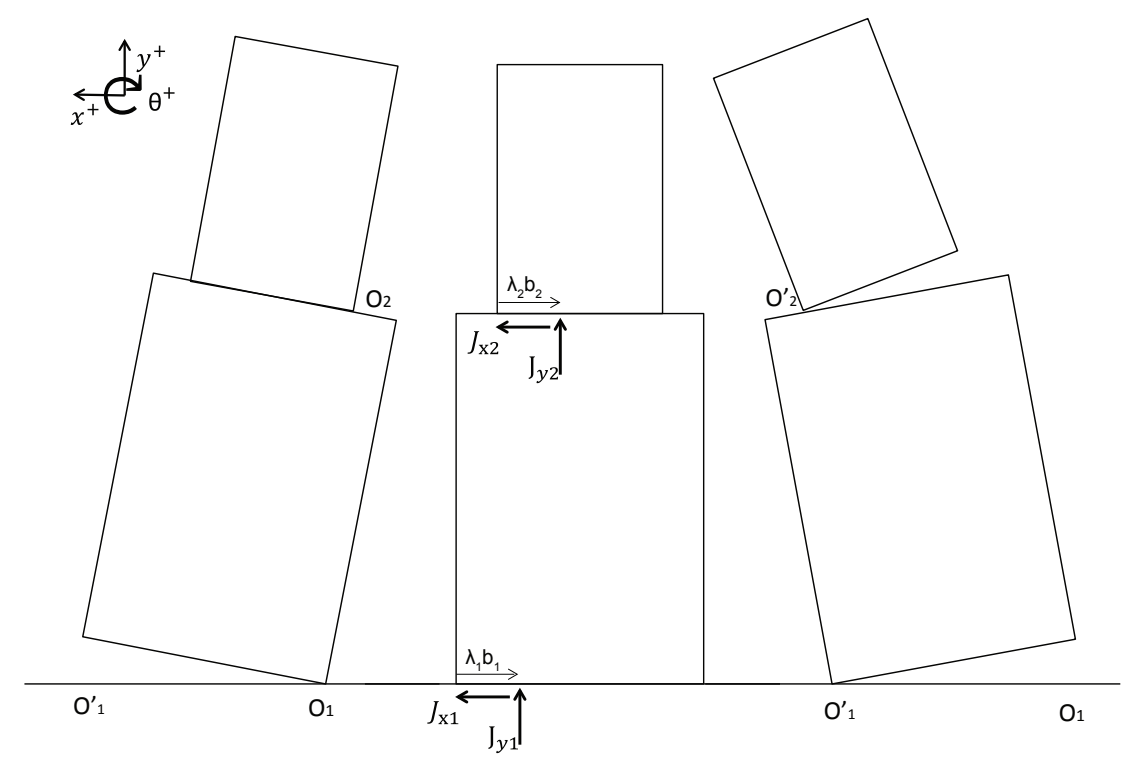

FIG. 8 - Transition from pattern $3 \mathrm{a}$ to $1 \mathrm{~b}$. 


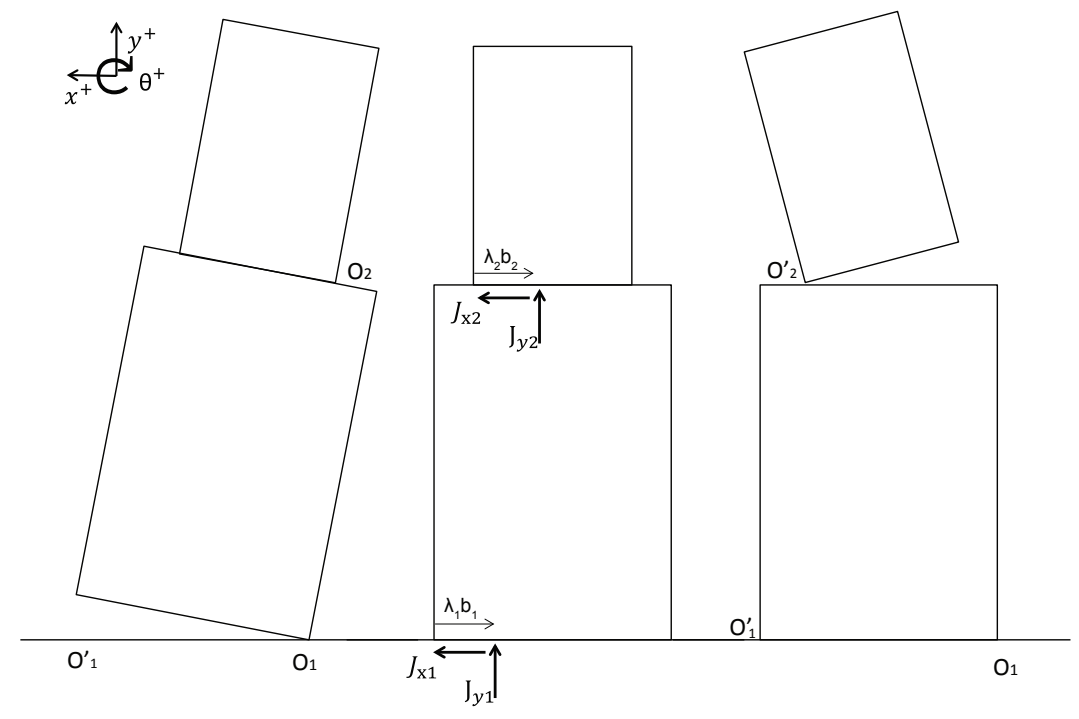

FIG. 9 - Transition from pattern 3a to 4 b. 


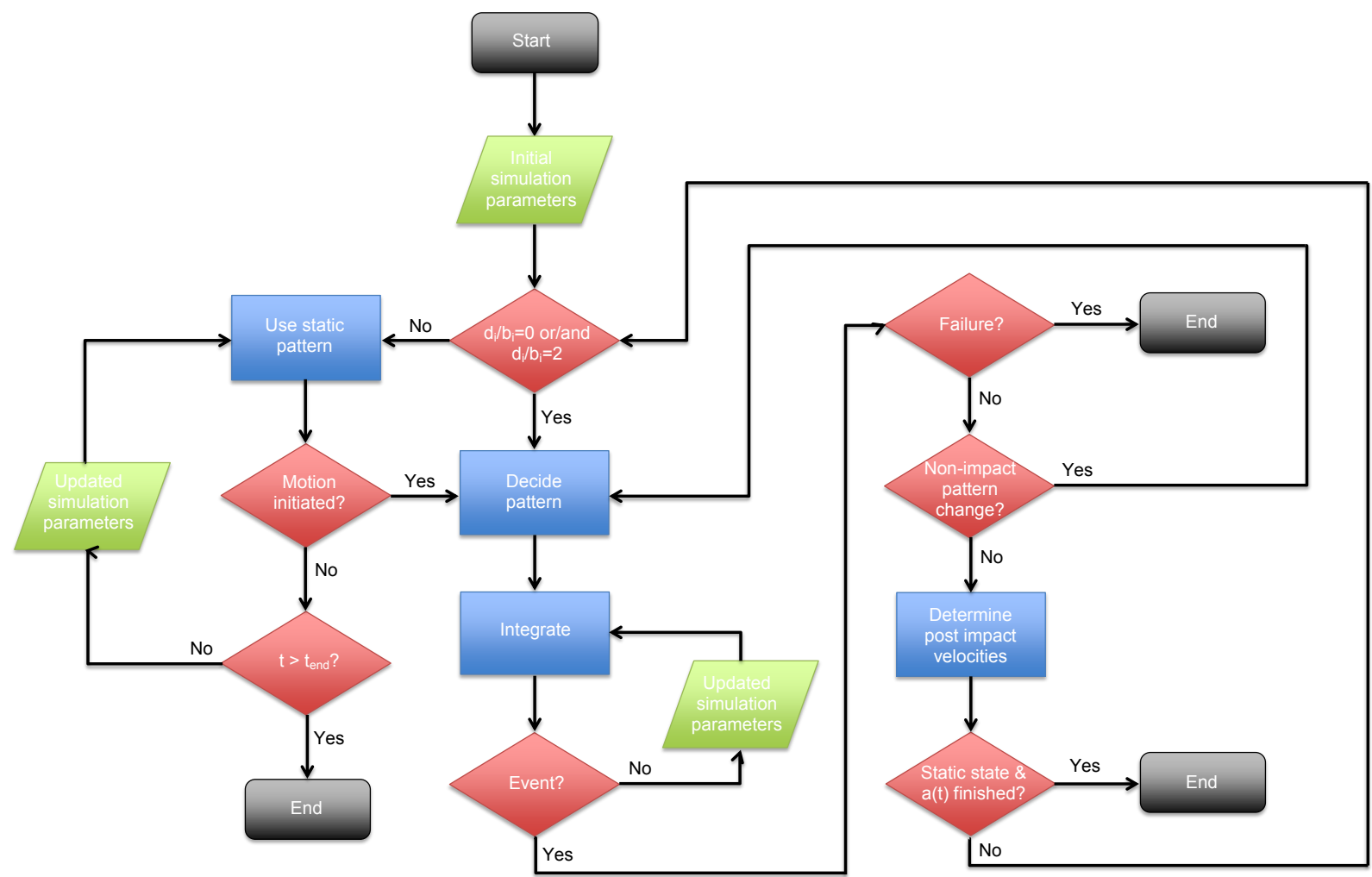

FIG. 10 - Flowchart to show simulation coding structure 


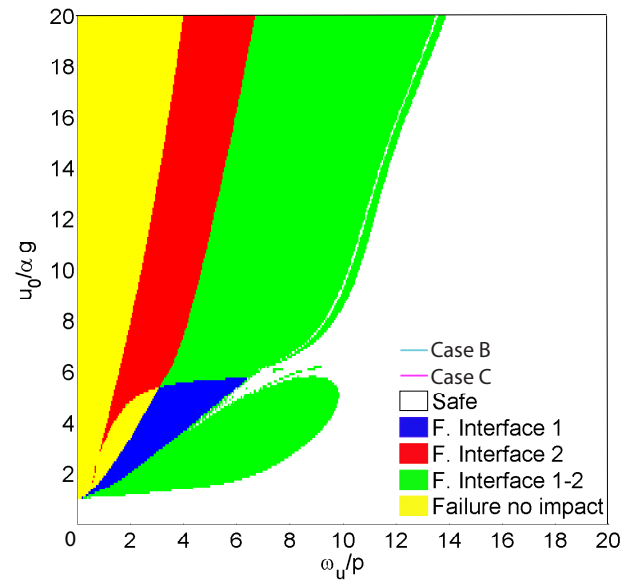

(a) History of impacts leading to failure

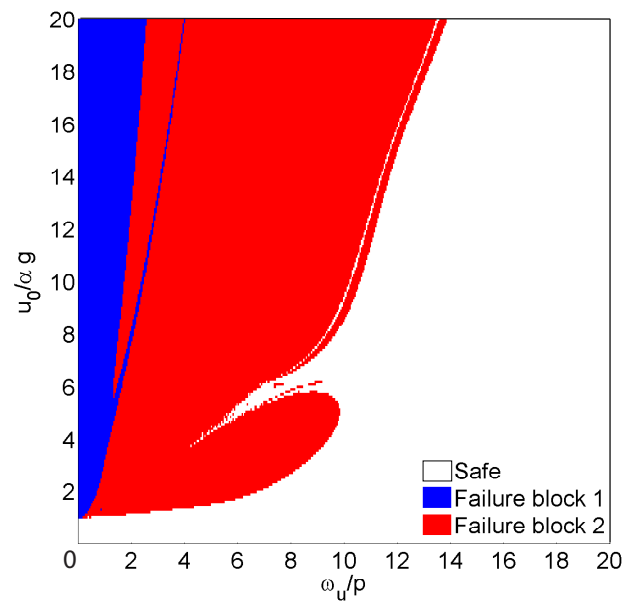

(b) Failure of upper and lower body

FIG. 11 - Diagrams of two stacked bodies with dimensions block 1: $h_{1}=0.6$, $b_{1}=0.2$ and block $2: h_{2}=0.15, b_{2}=0.05$ with $\lambda_{2}=0$ and $\lambda_{1}=0$. The horizontal and vertical axes are normalised with $p=6.8215$ and $\alpha=0.3218$. a) History of impacts leading to failure, b) Failure block 1 or block 2 . 


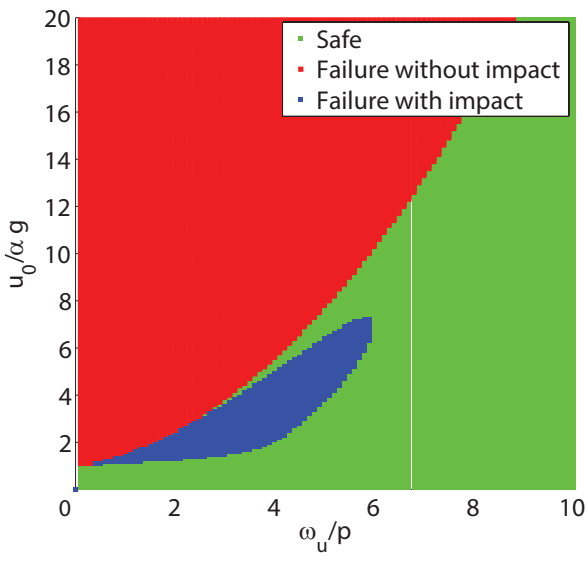

(a) Case B

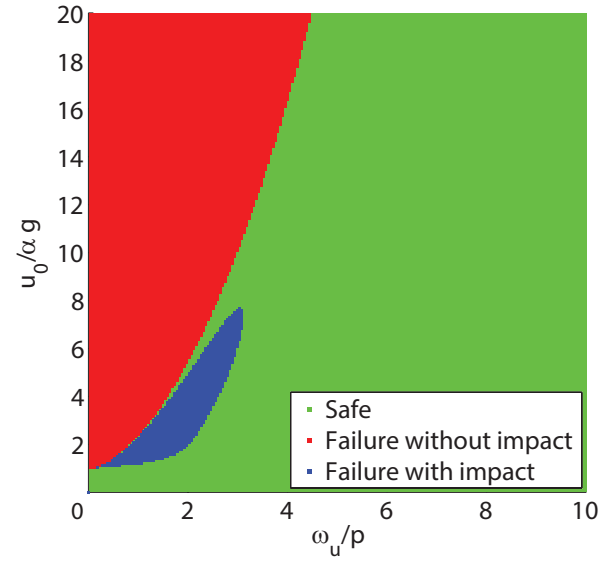

(b) Case C

FIG. 12 - Stability diagrams for a) case B b) case C. 


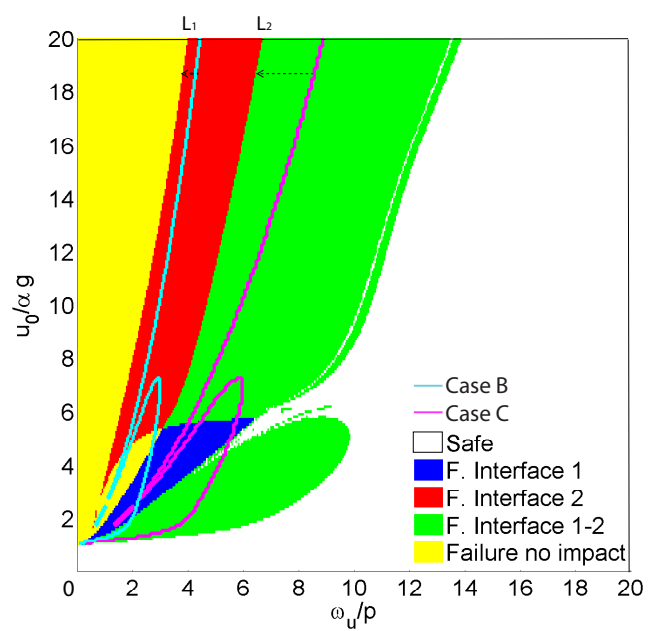

FIG. 13 - Comparison of stacked bodies failure with single bodies. Stacked bodies dimensions: $h_{1}=0.6, b_{1}=0.2$ and $h_{2}=0.15, b_{2}=0.05$. The dimensions for the single rocking cases: Block $1 h_{1}=0.6, b_{1}=0.2$ and Block $2 h_{1}=0.15$, $b_{1}=0.05$. The horizontal and vertical axes are normalised with $p=6.8215$ and $\alpha=0.3218$. Note that the single body case, only the boundaries of failure without impact are presented. It is assumed for all cases that $\lambda=\lambda_{i}=0$. 


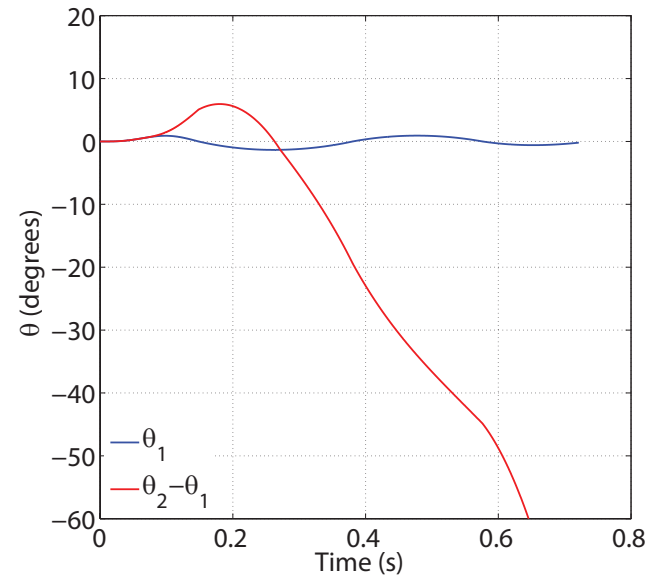

(a) Impact both bodies

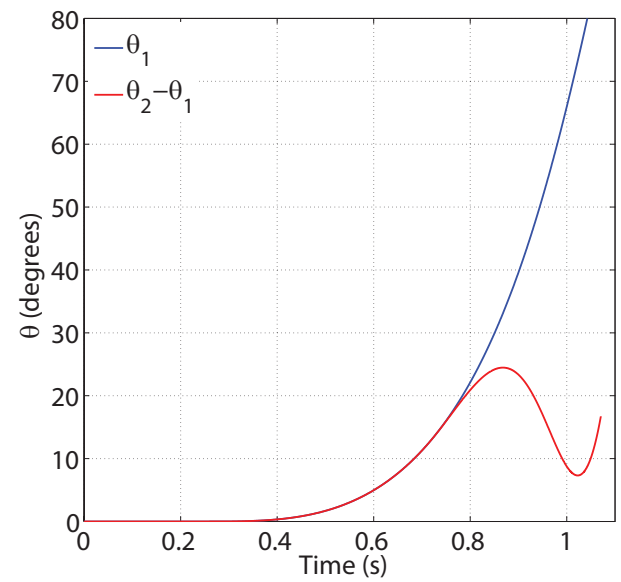

(c) Failure no impact

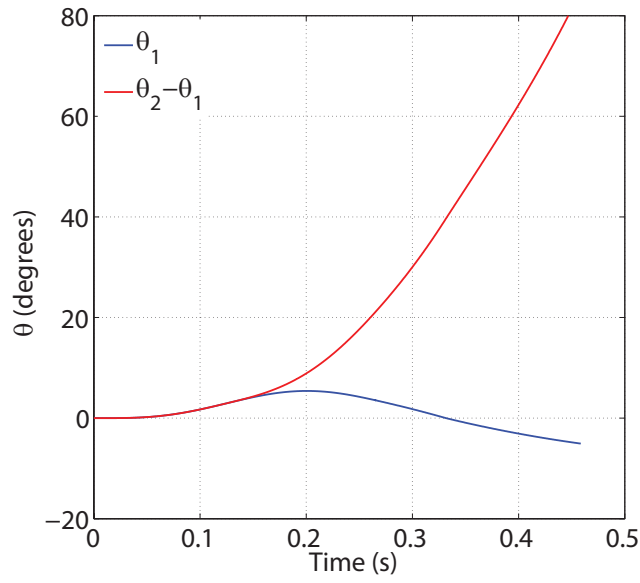

(b) Impact block 1

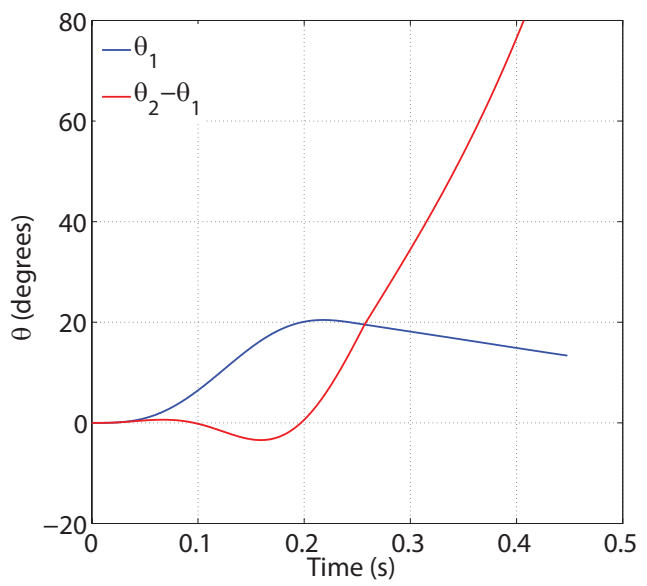

(d) Impact block 2

FIG. 14 - Time histories for a stacked bodies with dimensions $h_{1}=0.6, b_{1}=0.2$, $h_{2}=0.2, b_{2}=0.05$ with $\lambda_{1}=0$ and $\lambda_{2}=0$ for a) $\omega_{u} / p=2, u_{0} /(\alpha g)=1.81$, b) $\omega_{u} / p=2, u_{0} /(\alpha g)=2.4$, c) $\omega_{u} / p=2, u_{0} /(\alpha g)=4.01$ and d) $\omega_{u} / p=2$, $u_{0} /(\alpha g)=6.21$. 


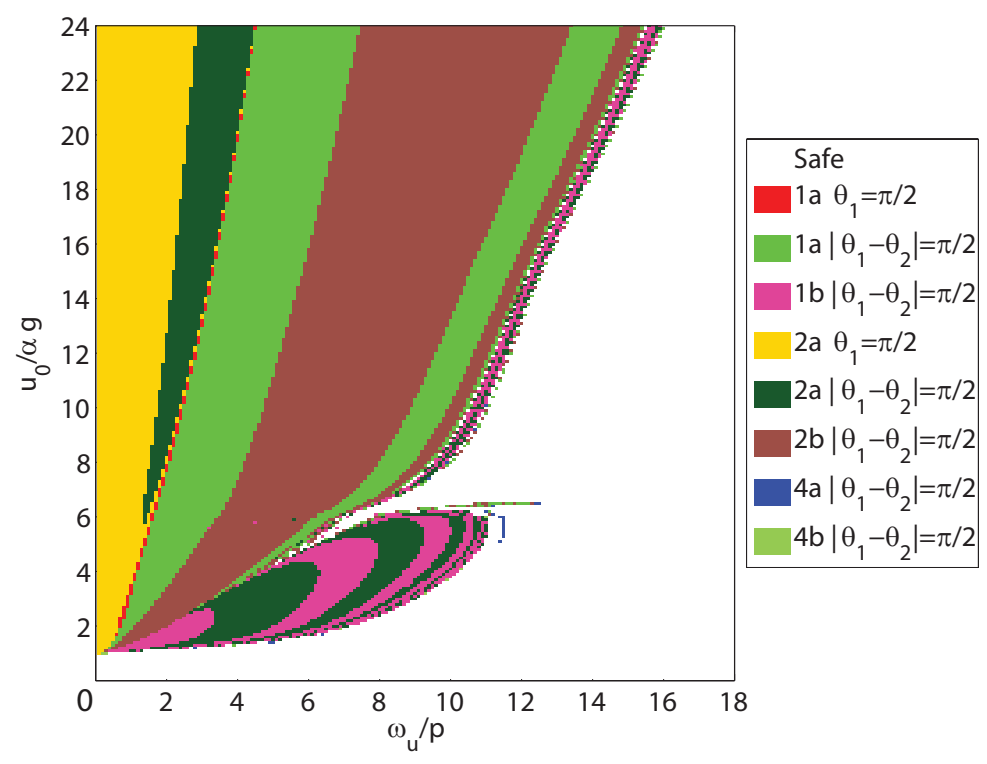

FIG. 15 - Rocking patterns of failure of stacked bodies with dimensions $h_{1}=0.6$, $b_{1}=0.2, h_{2}=0.15, b_{2}=0.05$ with $\lambda_{2}=0$ and $\lambda_{1}=0$. The horizontal and vertical axes are normalised with $p=6.8215$ and $\alpha=0.3218$. 


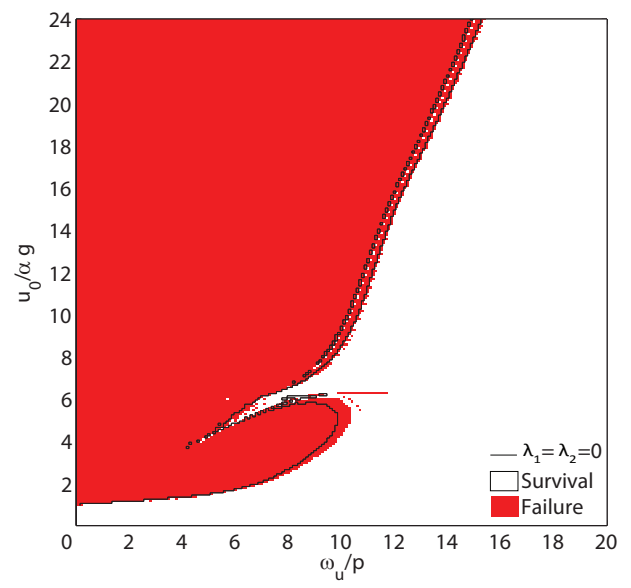

(a) $\lambda_{1}=0.3, \lambda_{2}=0$

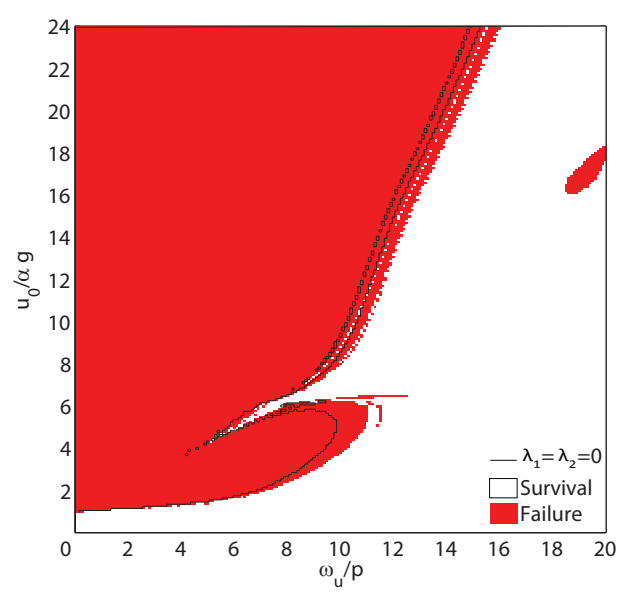

(c) $\lambda_{1}=0.3, \lambda_{2}=0.3$

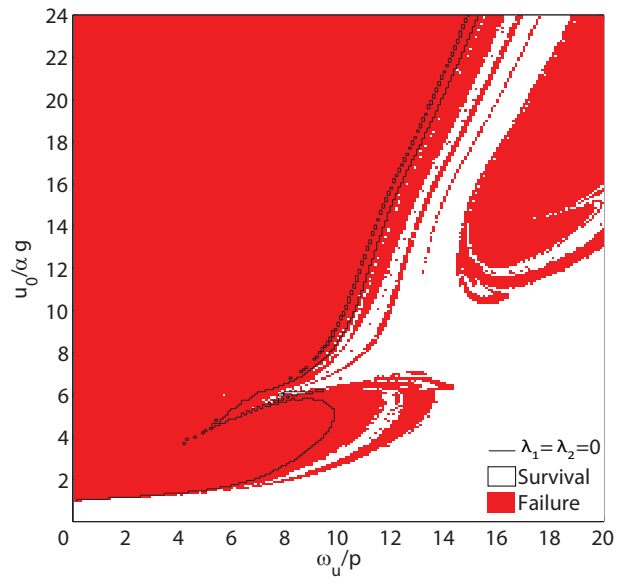

(b) $\lambda_{1}=0.9, \lambda_{2}=0$

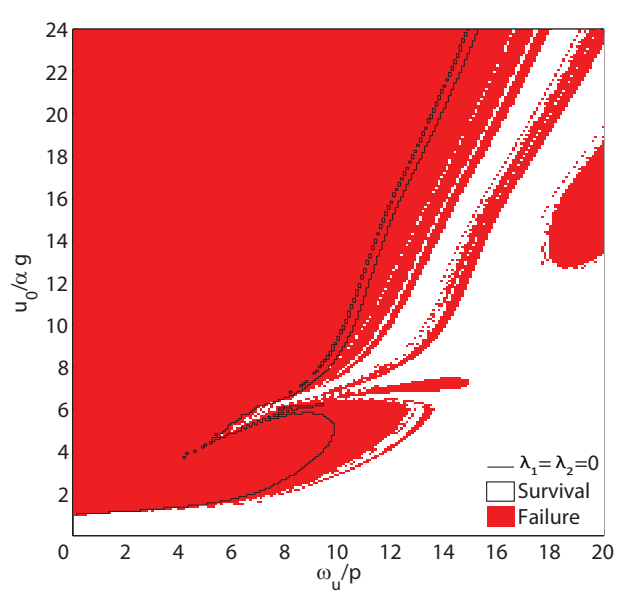

(d) $\lambda_{1}=0.3, \lambda_{2}=0.9$

FIG. 16 - Stability diagram of two stacked bodies with dimensions $h_{1}=0.6$, $b_{1}=0.2, h_{2}=0.15, b_{2}=0.05$ for a) $\lambda_{1}=0.3, \lambda_{2}=0$, b) $\lambda_{1}=0.9, \lambda_{2}=0$ c) $\left.\lambda_{1}=0.3, \lambda_{2}=0.3 \mathrm{~d}\right) \lambda_{1}=0.3, \lambda_{2}=0.9$. The horizontal and vertical axes are normalised with $p=6.8215$ and $\alpha=0.3218$. The boundaries between survival and failure when $\lambda_{1}=0, \lambda_{2}=0$ are plotted for explanation. 


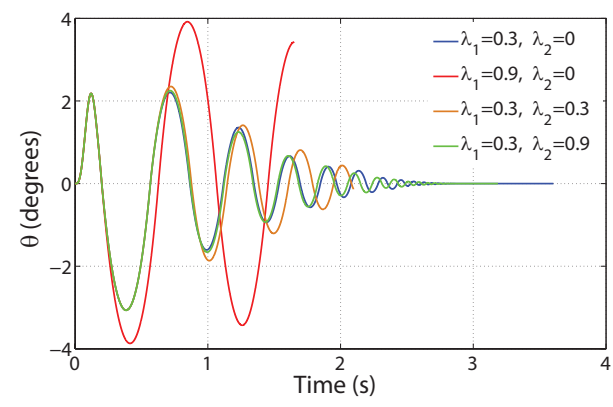

(a)

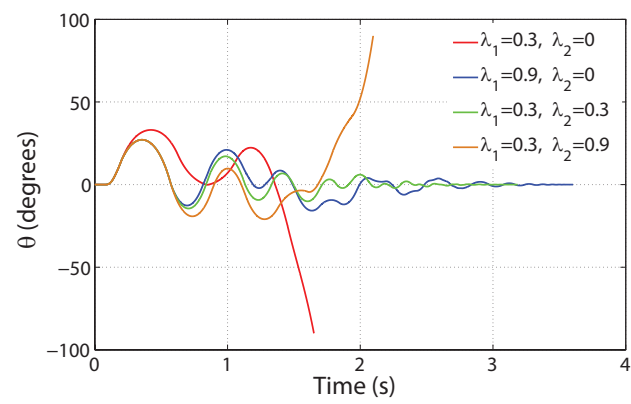

(b)

FIG. 17 - Time histories for a stacked bodies with dimensions $h_{1}=0.6, b_{1}=0.2$, $h_{2}=0.15, b_{2}=0.05$ when $\omega_{u} / p=12$ and $u_{0} /(\alpha g)=5.25$ for different values of $\lambda_{i}$ for a) $\theta_{1}$, b) $\theta_{2}-\theta_{1}$. 


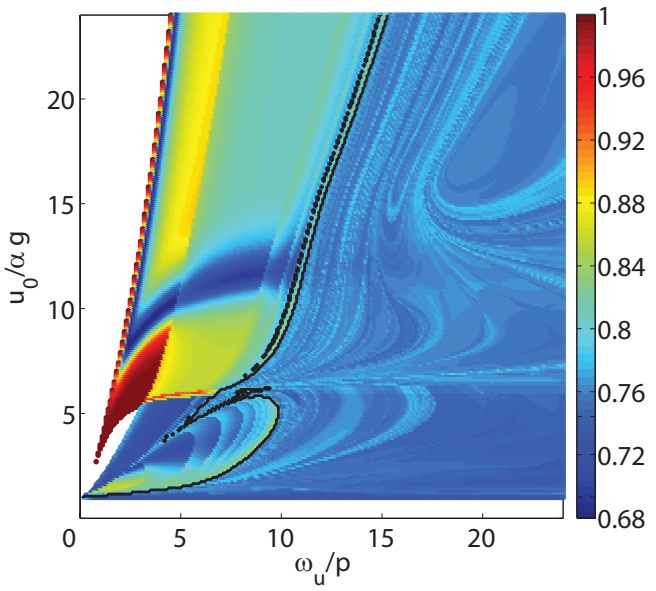

(a) $\lambda_{1}=0, \lambda_{2}=0$

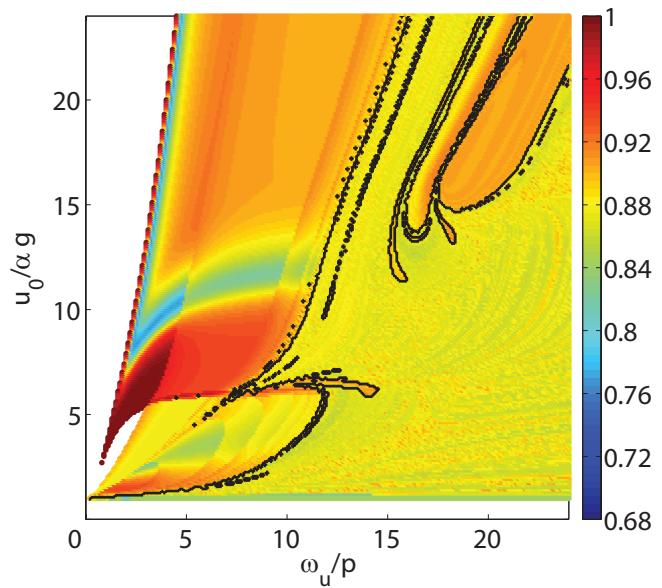

(b) $\lambda_{1}=0.6, \lambda_{2}=0.3$

FIG. 18 - Loss of energy factor $R_{t}$ for the system. The dimensions of the bodies are $h_{1}=0.6, b_{1}=0.2, h_{2}=0.2, b_{2}=0.05$ for a) $\lambda_{1}=0, \lambda_{2}=0$ and b) $\lambda_{1}=0.6$, $\lambda_{2}=0.3$. Boundaries between survival and failure for both cases are plotted for explanation. The horizontal and vertical axes are normalised with $p=6.8215$ and $\alpha=0.3218$. 


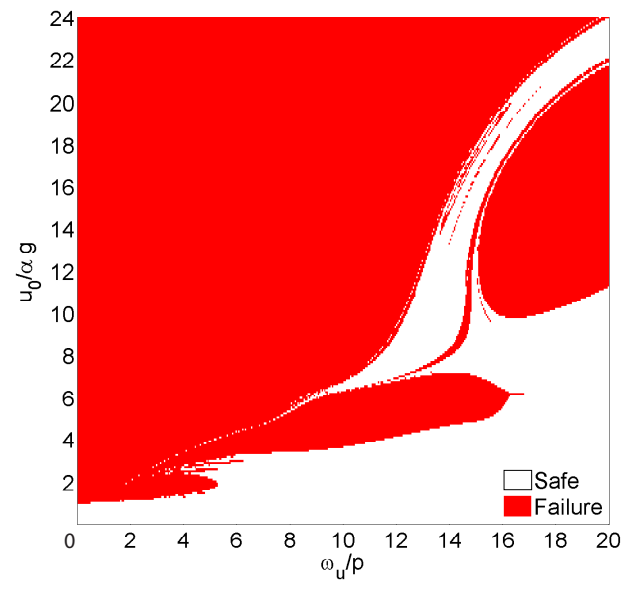

(a) $\lambda_{1}=0, \lambda_{2}=0$

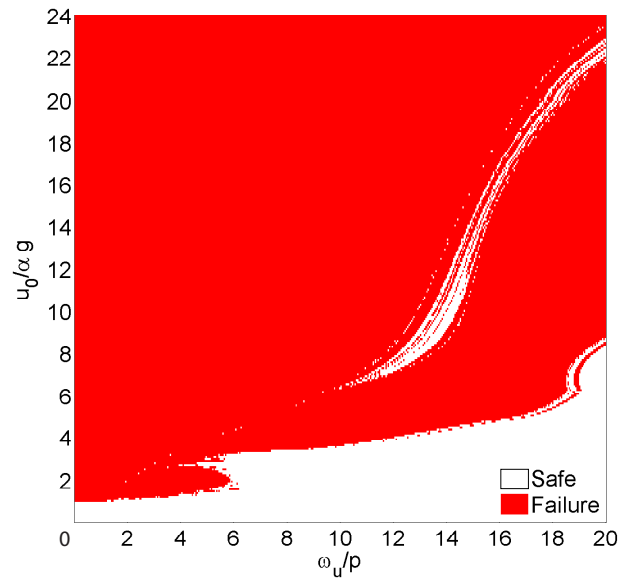

(b) $\lambda_{1}=0.6, \lambda_{2}=0.3$

FIG. 19 - Stability diagram of two stacked bodies subjected to two sinusoidal pulses. The dimensions of the bodies are $h_{1}=0.6, b_{1}=0.2, h_{2}=0.2, b_{2}=0.05$ for a) $\lambda_{1}=0, \lambda_{2}=0$ b) $\lambda_{1}=0.6, \lambda_{2}=0.3$. The horizontal and vertical axes are normalised with $p=6.8215$ and $\alpha=0.3218$. 


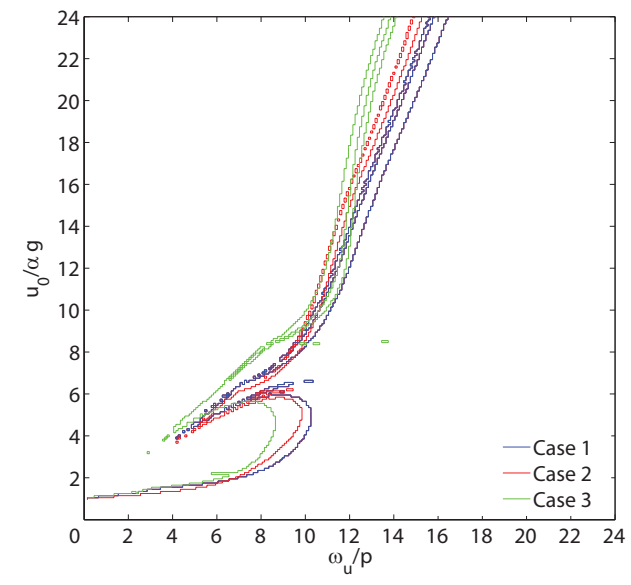

(a) $\lambda_{1}=0, \lambda_{2}=0$

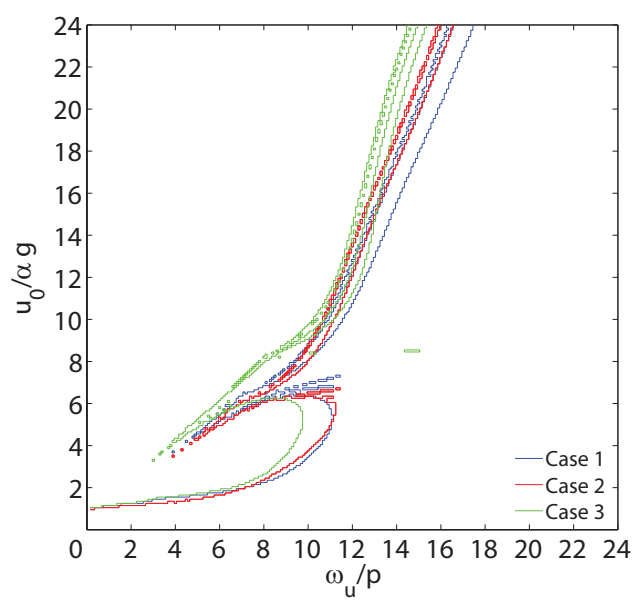

(c) $\lambda_{1}=0, \lambda_{2}=0.6$

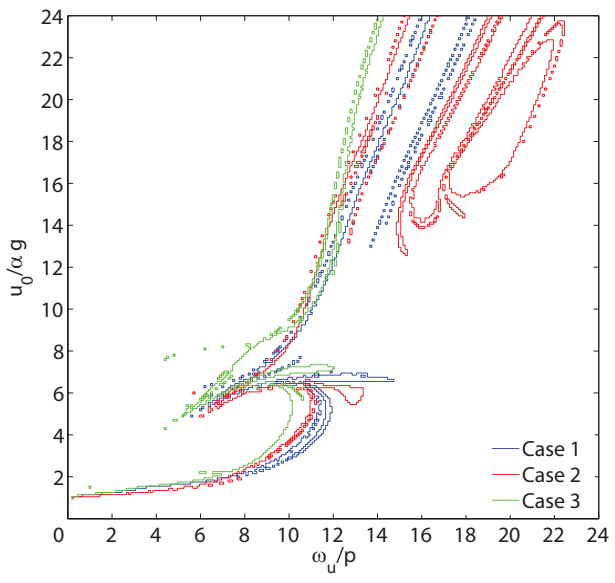

(b) $\lambda_{1}=0.6, \lambda_{2}=0$

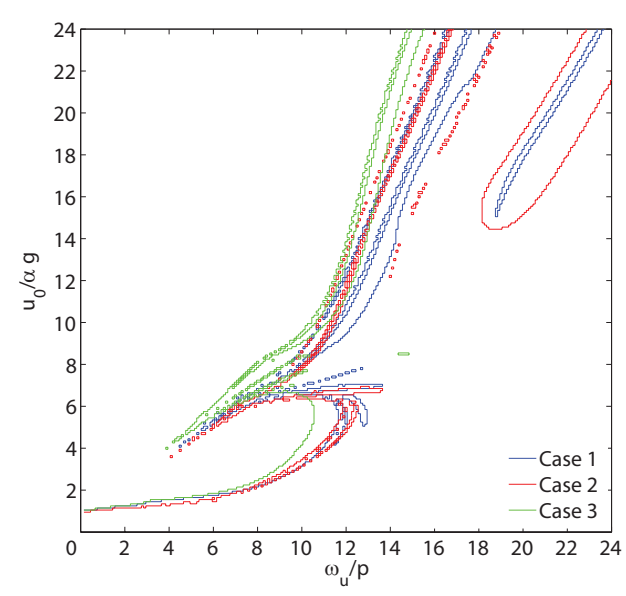

(d) $\lambda_{1}=0.3, \lambda_{2}=0.6$

FIG. 20 - Contour plots for a) $\lambda_{1}=\lambda_{2}=0$ b) $\lambda_{1}=0.6, \lambda_{2}=0$, c) $\lambda_{1}=0$, $\lambda_{2}=0.6$ and d) $\lambda_{1}=0.3, \lambda_{2}=0.6$ for Stacked Bodies 1, 2 and 3 defined in Table 2 . 


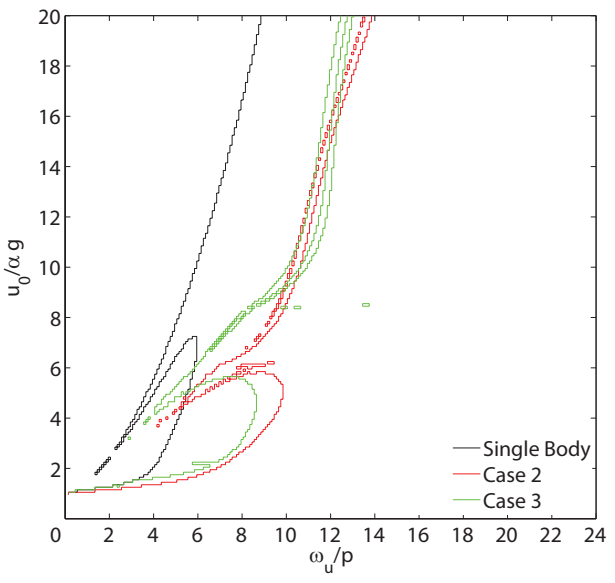

(a) $\lambda=\lambda_{1}=\lambda_{2}=0$

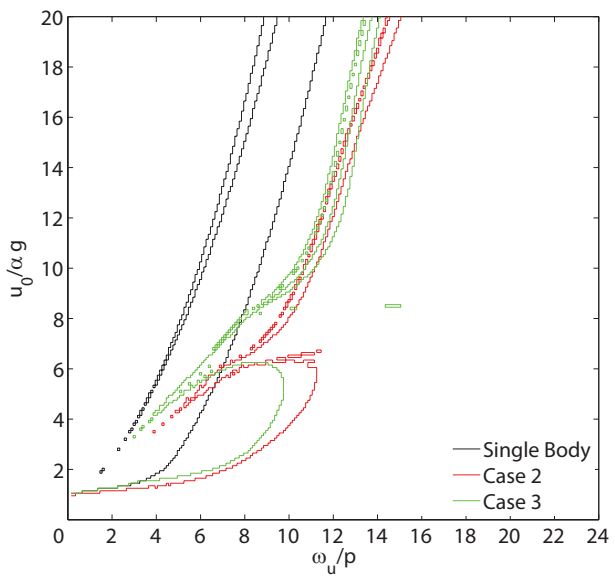

(b) $\lambda=\lambda_{2}=0.6, \lambda_{1}=0$

FIG. 21 - Comparison of stacked bodies cases 2 and 3 and block 2 acting as a single body when a) $\lambda=\lambda_{1}=\lambda_{2}=0$ and b) $\lambda=\lambda_{2}=0.6, \lambda_{1}=0$. 


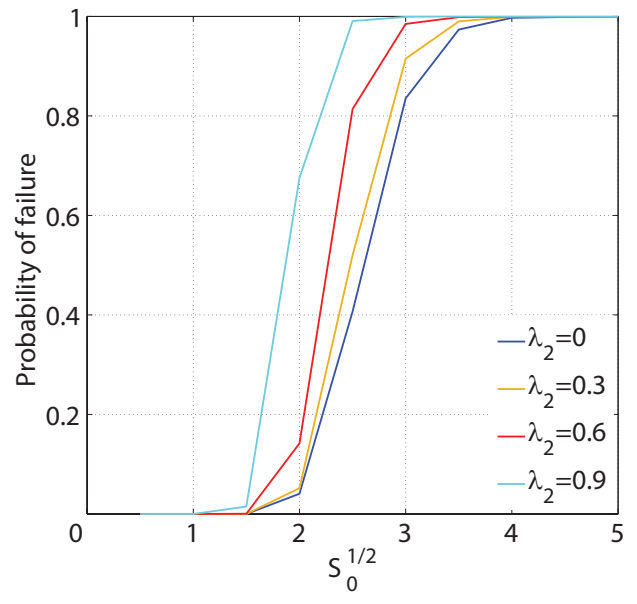

(a) $\lambda_{1}=0$

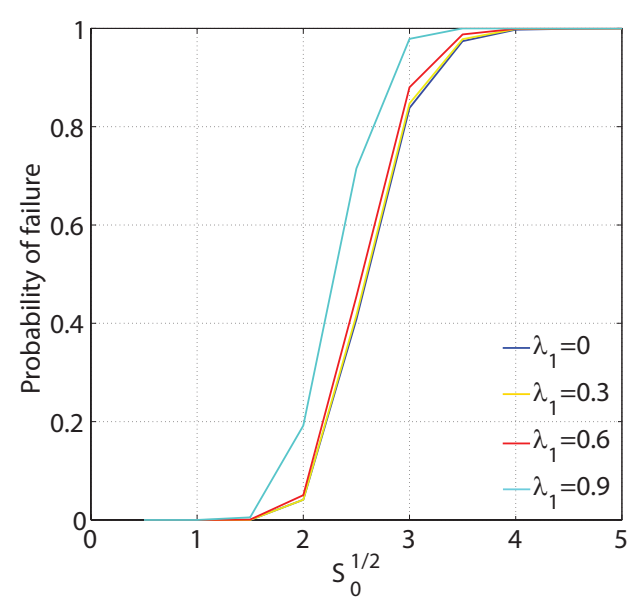

(c) $\lambda_{2}=0$

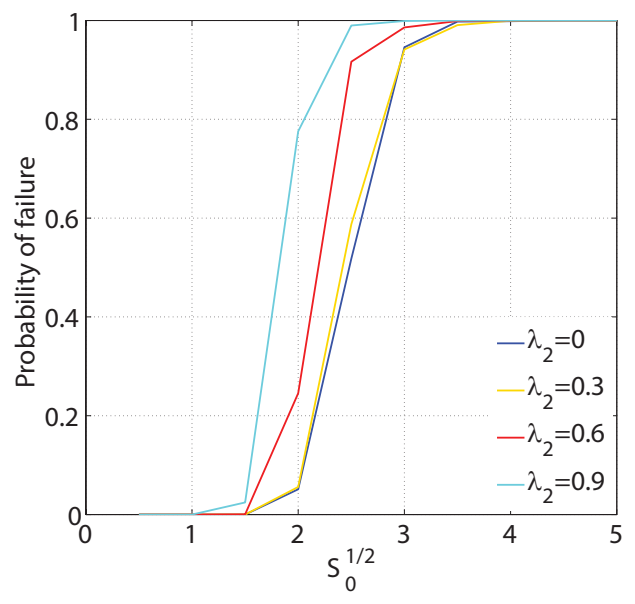

(b) $\lambda_{1}=0.3$

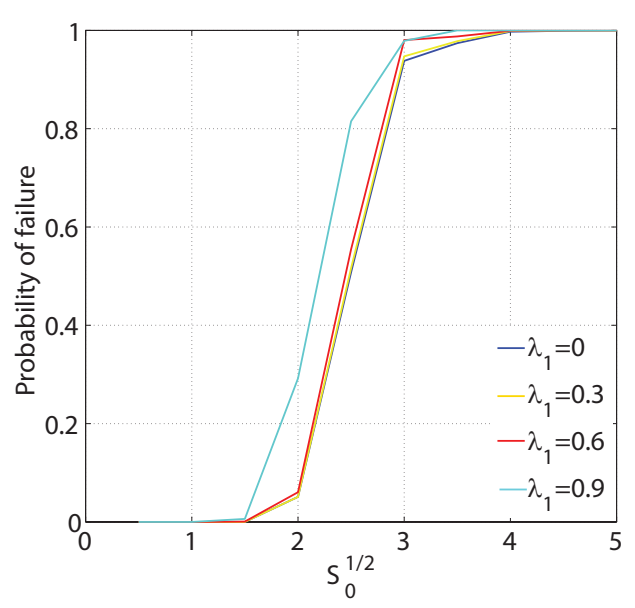

(d) $\lambda_{2}=0.3$

FIG. 22 - Fragility curves for different values of $\lambda_{2}$, when a) $\lambda_{1}=0$, b) $\lambda_{1}=0.3$, and $\lambda_{1}$ c) $\lambda_{2}=0$ and d) $\lambda_{2}=0.3$ for bodies with dimensions $h_{1}=0.5565$, $b_{1}=0.3970, h_{2}=0.3$ and $b_{2}=0.15$. 


\section{List of Tables}

1 Required interface conditions for each rocking pattern. . . . . . 54

2 Default transitions from pre-impact to post-impact pattern. . . . 55

3 Special conditions for switching patterns. PI refers to Post-Impact. 56

4 Dimensions for the different cases. . . . . . . . . . . . . . . 57 
TABLE 1 - Required interface conditions for each rocking pattern.

\begin{tabular}{ccc}
\hline Pattern & Bottom & Top \\
\hline Pattern 1a & $d_{1} / b_{1} \rightarrow 0$ & $d_{2} / b_{2} \rightarrow 0$ \\
Pattern 1b & $d_{1} / b_{1} \rightarrow 2$ & $d_{2} / b_{2} \rightarrow 2$ \\
Pattern 2a & $d_{1} / b_{1} \rightarrow 0$ & $d_{2} / b_{2} \rightarrow 2$ \\
Pattern 2b & $d_{1} / b_{1} \rightarrow 2$ & $d_{2} / b_{2} \rightarrow 0$ \\
Pattern 3a & $d_{1} / b_{1} \rightarrow 0$ & $d_{2} / b_{2} \in[0-2]$ \\
Pattern 3b & $d_{1} / b_{1} \rightarrow 2$ & $d_{2} / b_{2} \in[0-2]$ \\
Pattern 4a & $d_{1} / b_{1} \in[0-2]$ & $d_{2} / b_{2} \rightarrow 0$ \\
Pattern 4b & $d_{1} / b_{1} \in[0-2]$ & $d_{2} / b_{2} \rightarrow 2$ \\
\hline
\end{tabular}


TABLE 2 - Default transitions from pre-impact to post-impact pattern.

\begin{tabular}{|c|c|c|c|}
\hline Pattern & Switch & $P_{1}$ & $P_{2}$ \\
\hline $1 \mathrm{a}$ & $2 \mathrm{~b}$ & $O_{1}^{\prime} \stackrel{\lambda_{1} b_{1}}{\longrightarrow}$ & $P_{2}=O_{2}$ \\
\hline $1 \mathrm{a}$ & $2 \mathrm{a}$ & $P_{1}=O_{1}$ & $O_{2}^{\prime} \stackrel{\lambda_{2} b_{2}}{\longrightarrow}$ \\
\hline $1 b$ & $2 \mathrm{a}$ & $\stackrel{\lambda_{1} b_{1}}{\longleftarrow} O_{1}$ & $O_{2}^{\prime} \stackrel{\lambda_{2} b_{2}}{\longrightarrow}$ \\
\hline $1 b$ & $2 \mathrm{~b}$ & $P_{1}=O_{1}^{\prime}$ & $\stackrel{\lambda_{2} b_{2}}{\longleftarrow} O_{2}$ \\
\hline $2 \mathrm{a}$ & $1 b$ & $\stackrel{\lambda_{1} b_{1}}{\longleftarrow} O_{1}$ & $P_{2}=O_{2}^{\prime}$ \\
\hline $2 \mathrm{a}$ & $1 \mathrm{a}$ & $P_{1}=O_{1}$ & $\stackrel{\lambda_{2} b_{2}}{\longleftarrow} O_{2}$ \\
\hline $2 \mathrm{~b}$ & $1 \mathrm{a}$ & $\stackrel{\lambda_{1} b_{1}}{\longleftarrow} O_{1}$ & $P_{2}=O_{2}$ \\
\hline $2 \mathrm{~b}$ & $1 b$ & $P_{1}=O_{1}^{\prime}$ & $O_{2}^{\prime} \stackrel{\lambda_{2} b_{2}}{\longrightarrow}$ \\
\hline $3 a$ & $1 b$ & $O_{1}^{\prime} \stackrel{\lambda_{1} b_{1}}{\longrightarrow}$ & $O_{2}^{\prime} \stackrel{\lambda_{2} b_{2}}{\longrightarrow}$ \\
\hline $3 b$ & $1 \mathrm{a}$ & $\stackrel{\lambda_{1} b_{1}}{\longleftarrow} O_{1}$ & $\stackrel{\lambda_{2} b_{2}}{\longleftarrow} O_{2}$ \\
\hline $4 a$ & $1 b$ & $O_{1}^{\prime} \stackrel{\lambda_{1} b_{1}}{\longrightarrow}$ & $O_{2}^{\prime} \stackrel{\lambda_{2} b_{2}}{\longrightarrow}$ \\
\hline $4 \mathrm{~b}$ & $1 \mathrm{a}$ & $\stackrel{\lambda_{1} b_{1}}{\longleftarrow} O_{1}$ & $\stackrel{\lambda_{2} b_{2}}{\longleftarrow} O_{2}$ \\
\hline
\end{tabular}


TABLE 3 - Special conditions for switching patterns. PI refers to Post-Impact.

\begin{tabular}{|c|c|c|c|c|c|c|}
\hline Pattern & $\begin{array}{c}\text { Expected } \\
\text { Switch }\end{array}$ & $\begin{array}{c}\text { PI } \\
\text { condition }\end{array}$ & $\begin{array}{c}\text { New PI } \\
\text { condition }\end{array}$ & Switch & $P_{1}$ & $P_{2}$ \\
\hline $1 \mathrm{a}$ & $2 \mathrm{~b}$ & $\dot{\theta}_{1}^{+} \geq 0$ & $\dot{\theta}_{1}^{+}=0$ & $4 a$ & $O_{1}^{\prime} \stackrel{\lambda_{1} b_{1}}{\longrightarrow}$ & $P_{2}=O_{2}$ \\
\hline $1 \mathrm{a}$ & $2 \mathrm{a}$ & $\dot{\theta}_{2} \geq \dot{\theta}_{1}$ & $\dot{\theta}_{2}=\dot{\theta}_{1}$ & $3 a$ & $P_{1}=O_{1}$ & $O_{2}^{\prime} \stackrel{\lambda_{2} b_{2}}{\longrightarrow}$ \\
\hline $1 b$ & $2 \mathrm{a}$ & $\dot{\theta}_{1}^{+} \leq 0$ & $\dot{\theta}_{1}^{+}=0$ & $4 \mathrm{~b}$ & $\stackrel{\lambda_{1} b_{1}}{\longleftarrow} O_{1}$ & $P_{2}=O_{2}^{\prime}$ \\
\hline $1 b$ & $2 \mathrm{~b}$ & $\dot{\theta}_{2} \leq \dot{\theta}_{1}$ & $\dot{\theta}_{2}=\dot{\theta}_{1}$ & $3 \mathrm{~b}$ & $P_{1}=O_{1}^{\prime}$ & $\stackrel{\lambda_{2} b_{2}}{\longleftarrow} O_{2}$ \\
\hline $2 \mathrm{a}$ & $1 b$ & $\dot{\theta}_{1}^{+} \geq 0$ & $\dot{\theta}_{1}^{+}=0$ & $4 \mathrm{~b}$ & $O_{1}^{\prime} \stackrel{\lambda_{1} b_{1}}{\longrightarrow}$ & $P_{2}=O_{2}^{\prime}$ \\
\hline $2 \mathrm{a}$ & $1 \mathrm{a}$ & $\dot{\theta}_{2}^{+} \geq \dot{\theta}_{1}^{+}$ & $\dot{\theta}_{2}^{+}=\dot{\theta}_{1}^{+}$ & $3 a$ & $P_{1}=O_{1}$ & $O_{2}^{\prime} \stackrel{\lambda_{2} b_{2}}{\longrightarrow}$ \\
\hline $2 b$ & $1 \mathrm{a}$ & $\dot{\theta}_{1}^{+} \leq 0$ & $\dot{\theta}_{1}^{+}=0$ & $4 a$ & $\stackrel{\lambda_{1} b_{1}}{\longleftarrow} O_{1}$ & $P_{2}=O_{2}$ \\
\hline $2 \mathrm{~b}$ & $1 b$ & $\dot{\theta}_{2}^{+} \leq \dot{\theta}_{1}^{+}$ & $\dot{\theta}_{2}^{+}=\dot{\theta}_{1}^{+}$ & $3 \mathrm{~b}$ & $P_{1}=O_{1}^{\prime}$ & $\stackrel{\lambda_{2} b_{2}}{\longleftarrow} O_{2}$ \\
\hline $3 \mathrm{a}$ & $1 b$ & $\dot{\theta}_{2}^{+} \geq \dot{\theta}_{1}^{+}$ & $\dot{\theta}_{2}^{+}=\dot{\theta}_{1}^{+}$ & $3 b$ & $O_{1}^{\prime} \stackrel{\lambda_{1} b_{1}}{\longrightarrow}$ & $O_{2}^{\prime} \stackrel{\lambda_{2} b_{2}}{\longrightarrow}$ \\
\hline $3 a$ & $1 b$ & $\dot{\theta}_{1}^{+} \geq 0$ & $\dot{\theta}_{1}^{+}=0$ & $4 \mathrm{~b}$ & $O_{1} \stackrel{\lambda_{1} b_{1}}{\longrightarrow}$ & $\mathrm{O}_{2}^{\prime} \stackrel{\lambda_{2} b_{2}}{\longrightarrow}$ \\
\hline $3 \mathrm{~b}$ & $1 \mathrm{a}$ & $\dot{\theta}_{2}^{+} \leq \dot{\theta}_{1}^{+}$ & $\dot{\theta}_{2}^{+}=\dot{\theta}_{1}^{+}$ & $3 a$ & $\stackrel{\lambda_{1} b_{1}}{\longleftarrow} O_{1}$ & $\stackrel{\lambda_{2} b_{2}}{\longleftarrow} O_{2}$ \\
\hline $3 b$ & $1 \mathrm{a}$ & $\dot{\theta}_{1}^{+} \leq 0$ & $\dot{\theta}_{1}^{+}=0$ & $4 a$ & $\stackrel{\lambda_{1} b_{1}}{\longleftarrow} O_{1}$ & $\stackrel{\lambda_{2} b_{2}}{\longleftarrow} O_{2}$ \\
\hline $4 \mathrm{a}$ & $1 b$ & $\dot{\theta}_{1}^{+} \geq 0$ & $\dot{\theta}_{1}^{+}=0$ & $4 \mathrm{~b}$ & $O_{1}^{\prime} \stackrel{\lambda_{1} b_{1}}{\longrightarrow}$ & $O_{2}^{\prime} \stackrel{\lambda_{2} b_{2}}{\longrightarrow}$ \\
\hline $4 \mathrm{a}$ & $1 b$ & $\dot{\theta}_{1}^{+} \leq 0, \dot{\theta}_{2}^{+}>\dot{\theta}_{1}^{+}$ & $\dot{\theta}_{2}^{+}=\dot{\theta}_{1}^{+}$ & $3 \mathrm{~b}$ & $O_{1}^{\prime} \stackrel{\lambda_{1} b_{1}}{\longrightarrow}$ & $O_{2}^{\prime} \stackrel{\lambda_{2} b_{2}}{\longrightarrow}$ \\
\hline $4 \mathrm{~b}$ & $1 \mathrm{a}$ & $\dot{\theta}_{1}^{+} \leq 0$ & $\dot{\theta}_{1}^{+}=0$ & $4 a$ & $\stackrel{\lambda_{1} b_{1}}{\longleftarrow} O_{1}$ & $\stackrel{\lambda_{2} b_{2}}{\longleftarrow} O_{2}$ \\
\hline $4 b$ & $1 \mathrm{a}$ & $\dot{\theta}_{1}^{+} \geq 0, \dot{\theta}_{2}^{+}<\dot{\theta}_{1}^{+}$ & $\dot{\theta}_{2}^{+}=\dot{\theta}_{1}^{+}$ & $3 \mathrm{a}$ & $\stackrel{\lambda_{1} b_{1}}{\longleftarrow} O_{1}$ & $\stackrel{\lambda_{2} b_{2}}{\longleftarrow} O_{2}$ \\
\hline
\end{tabular}


TABLE 4 - Dimensions for the different cases.

\begin{tabular}{lcccccc}
\hline & \multicolumn{3}{c}{ Body 1 } & \multicolumn{3}{c}{ Body 2 } \\
\hline & $h_{1}[\mathrm{~cm}]$ & $b_{1}[\mathrm{~cm}]$ & $h_{1} / b_{1}$ & $h_{2}[\mathrm{~cm}]$ & $b_{2}[\mathrm{~cm}]$ & $h_{2} / b_{2}$ \\
\hline Case 1 & 60 & 20 & 3 & 20 & 5 & 4 \\
Case 2 & 60 & 20 & 3 & 15 & 5 & 3 \\
Case 3 & 45 & 20 & 2.25 & 15 & 5 & 3 \\
\hline
\end{tabular}

